\title{
The molecular environment of NGC 3603
}

\section{Spatial distribution and kinematic structure ${ }^{\star}$}

\author{
D. E. A. Nürnberger ${ }^{1,2}$, L. Bronfman ${ }^{3}$, H. W. Yorke $^{4}$, and H. Zinnecker ${ }^{5}$ \\ 1 Institut für Theoretische Physik und Astrophysik, Univ. Würzburg, Am Hubland, 97074 Würzburg, Germany \\ 2 Institut de Radio-Astronomie Millimétrique, 300 rue de la Piscine DU, 38406 St. Martin-d'Hères, France \\ 3 Departamento de Astronomía, Universidad de Chile, Casilla 36 - D, Santiago, Chile \\ 4 Jet Propulsion Laboratory, California Inst. of Technology, 4800 Oak Grove Drive, Pasadena, CA 91109, USA \\ 5 Astrophysikalisches Institut Potsdam, An der Sternwarte 16, 14482 Potsdam, Germany
}

\section{Received 23 April 2002 / Accepted 9 July 2002}

\begin{abstract}
We present CS (2-1) and CS (3-2) observations of the molecular gas associated with the Galactic starburst template NGC 3603 , over an area of 5!8 $\times 16 ! 7$, with the OB cluster at the center. Total velocity integrated maps and channel maps give insight into the spatial distribution and the kinematic structure of the dense gas in the giant molecular cloud from which the starburst cluster originated.

We identify 13 molecular clumps with radii less than $0.8 \mathrm{pc}$ and derive upper limits for their virial masses as well as lower limits for their $\mathrm{H}_{2}$ column densities: $\left\langle\mathcal{M}_{\text {vir }}\right\rangle \lesssim(1.0 \pm 0.6) \times 10^{3} \mathcal{M}_{\odot}$ and $\left\langle\mathcal{N}\left(\mathrm{H}_{2}\right)\right\rangle \gtrsim(0.4 \pm 0.2) \times 10^{23} \mathrm{~cm}^{-2}$. One of the clumps, MM 11 , clearly stands out with a mass and column density 4 times higher than average. The CS (3-2)/CS (2-1) map shows higher intensity ratios to the south of the OB cluster than to the north $(0.80 \pm 0.08$ versus $0.32 \pm 0.11)$, which indicates a substantial difference in the physical conditions (either opacities or excitation temperatures) of the molecular gas. From the average of the line peak velocities, $14.2 \pm 1.6 \mathrm{~km} \mathrm{~s}^{-1}$, we deduce a kinematic distance of $7.7 \pm 0.2 \mathrm{kpc}$ for NGC 3603 .

We estimate the star formation efficiency $(\gtrsim 30 \%)$ of the central part of the NGC $3603 \mathrm{H}$ II region. If we assume the age of the $\mathrm{OB}$ cluster to be less than $3 \mathrm{Myr}$ and the star formation rate to be larger than $1.3 \times 10^{-3} \mathcal{M}_{\odot} \mathrm{yr}^{-1}$, the derived timescale for gas removal $(\tau \sim 6 \mathrm{Myr})$ can explain why the starburst cluster itself is nearly void of interstellar material. The remnant clump MM 1 appears to constitute the head of a prominent pillar which still becomes dispersed by ionizing radiation and stellar winds originating from the massive stars of the cluster. Because some of the molecular clumps are associated with near and mid infrared sources as well as $\mathrm{OH}, \mathrm{H}_{2} \mathrm{O}$ and $\mathrm{CH}_{3} \mathrm{OH}$ maser sources we conclude that star formation is still going on within NGC 3603.
\end{abstract}

Key words. stars: formation - ISM: clouds - H I regions - ISM: individual objects: NGC 3603 - ISM: kinematics and dynamics - ISM: structure

\section{Introduction}

It is well established that star formation occurs in giant molecular clouds (GMCs; Blitz \& Williams 1999; Bronfman et al. 2000; Williams et al. 2000). These GMCs may have masses up to $10^{6} M_{\odot}$, mean diameters of about $50 \mathrm{pc}$ and mean column densities of $5 \times 10^{21} \mathrm{~cm}^{-2}$ (e.g. Blitz 1993; Williams et al. 2000). According to Williams \& McKee (1997) a sharp cutoff at the upper end of the cloud mass distribution at about $6 \times 10^{6} M_{\odot}$ indicates that cloud masses might be either

Send offprint requests to: D. E. A. Nürnberger, e-mail: nurnberg@astro.uni-wuerzburg.de

* Based on data sets obtained at the European Southern Observatory, La Silla, Chile under proposal numbers 56.C-0670, 58.C-0662 and 60.C-0489. limited by the tidal field of the Galaxy or the disrupting effect of high mass stars $\left(\mathcal{M}>8 \mathcal{M}_{\odot}\right)$ within them.

Although relatively short-lived and scarce in comparison to their low mass counterparts, young OB stars emit a large amount of hydrogen-ionizing Lyman-Continuum (LyC) photons $-h v>13.6 \mathrm{eV}$ - and are associated both with high energetic winds and massive molecular outflows. Hence, they give rise to both the formation of (ultracompact) $\mathrm{H}$ II regions (e.g. Hollenbach et al. 1994; Yorke \& Welz 1996) and to the rapid destruction of their natal molecular environment.

By comparison of the distributions of Galactic giant molecular clouds with that of OB associations, Williams \& McKee (1997) have investigated the likelyhood of massive star formation in a cloud. They find that - on average - at least one star of spectral type $\mathrm{O}$ is contained within $10^{5} \mathcal{M}_{\odot}$ of gas. Although 


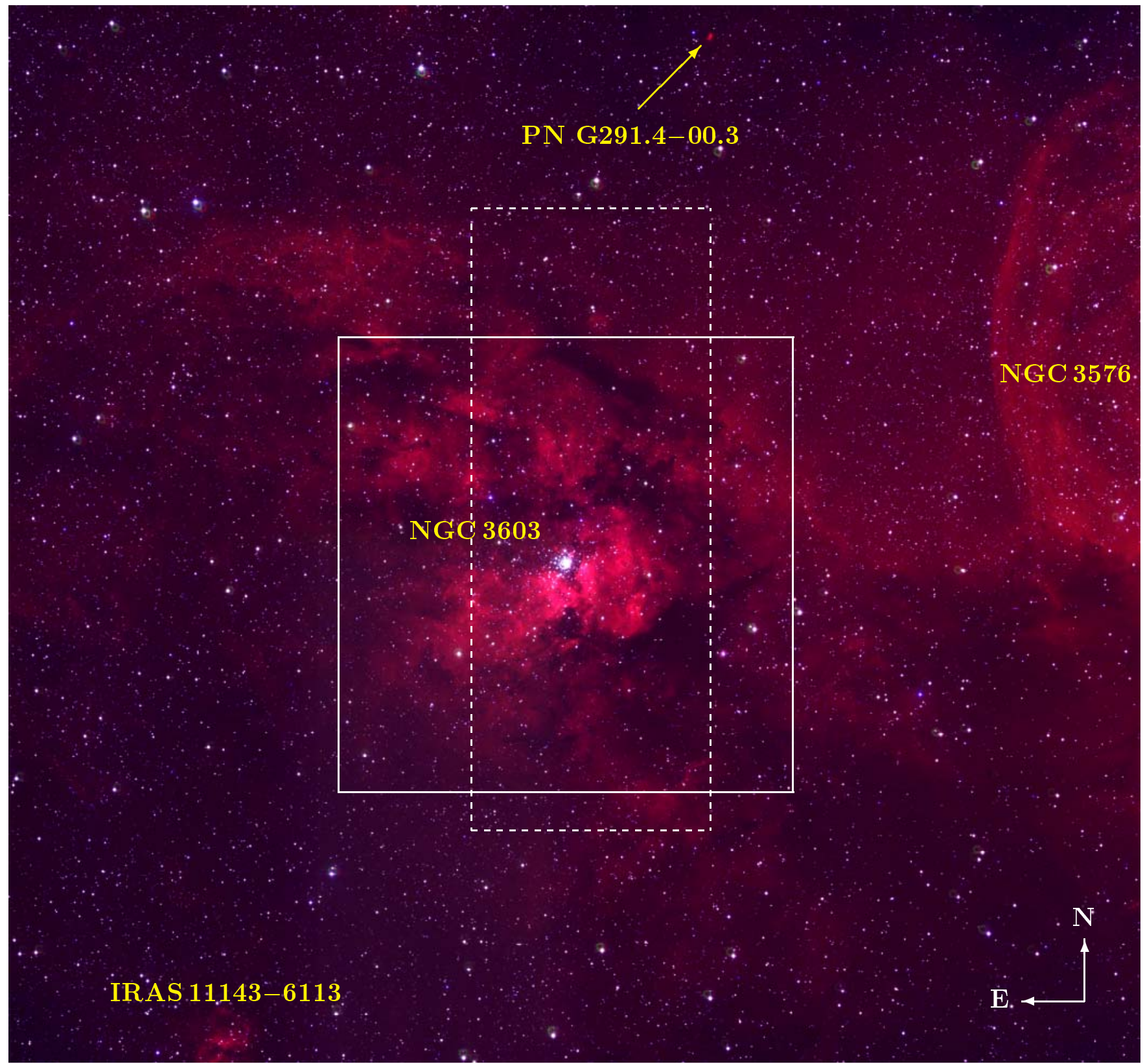

Fig. 1. Wide field image of the 33:7 × 31'.4 region centered on NGC 3603. The three-colour image combines observations taken in $\mathrm{H} \alpha(\mathrm{red})$, [S II] (green) and $8160 \AA$ A continuum (blue) filters and was obtained by Th. Stanke with the MPIA WFI mounted on the ESO/MPG $2.2 \mathrm{~m}$ telescope. The square outlines the central quarter as presented in Fig. 9. The area covered by our SEST observations and displayed in succeeding CS maps is indicated by the dashed box. Large parts of the image are dominated by diffuse emission from ionized material, which is associated with either NGC 3603 or NGC 3576 (Georgelin et al. 2000) and located at distances of $\sim 7 \mathrm{kpc}$ and $\sim 3 \mathrm{kpc}$, respectively (De Pree et al. 1999). Particularly, both $\mathrm{H}$ in regions are in sharp contrast to opaque clouds of cold gas and dust in the foreground. At the lower left one finds a third $\mathrm{H}$ II region primarily excited by the luminous IRAS $11143-6113$ source (distance $~ 8.5 \mathrm{kpc}$; Caswell \& Haynes 1987). A so far unknown PN was discovered near the northern edge of the image (see Nürnberger et al. 2001).

it may vary by more than 2 orders of magnitude and highly depend on the physical properties of individual clouds (in particular, their kinetic support by turbulence and magnetic fields), the average efficiency of star formation out of molecular material appears to be about 5-10\% (compare also to the values given by Tachihara et al. 2002) over the typical lifetime of OB associations. Taking into account typical timescales for photoevaporation, one can expect that GMCs of $10^{6} \mathcal{M}_{\odot}$ survive for about $3 \times 10^{7} \mathrm{yr}$.
From studies of the nearest regions which are showing evidence of high mass star formation (with Orion being the paradigm; Hillenbrand 1997) it is well known that massive stars preferentially form in clusters (see the reviews by Lada et al. 1993; Zinnecker et al. 1993; Elmegreen et al. 2000). Therefore, within an individual cloud, properties like star formation efficiency and lifetime of the GMC are supposed to significantly deviate from the average values given above. Such a scenario on small scales - compared to the whole extent of a GMC - 

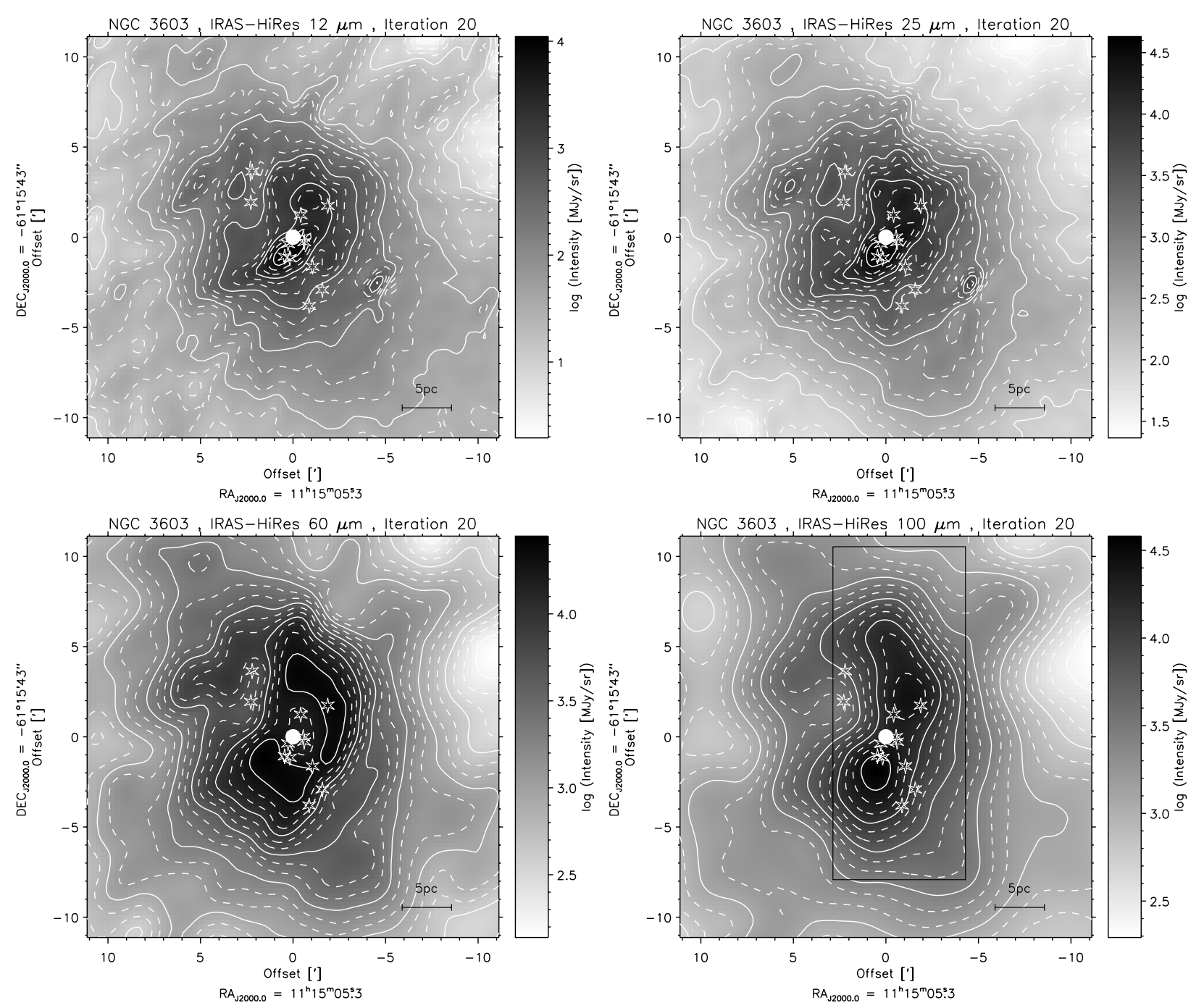

Fig. 2. IRAS-HiRes images of NGC 3603. The OB cluster is marked by the dot, some prominent IR sources of Frogel et al. (1977) are indicated by asterisks. The box outlines the area covered by our CS data.

immediately proposes an ongoing process, where the formation of massive stars is triggered successively in several spatially distinct generations.

Because only little is known about the triggering mechanisms of star formation progressing within GMCs we are carrying on a multi-wavelength study to investigate the interplay between the NGC 3603 OB cluster and its adjacent giant molecular cloud. Apart from the millimeter study presented here, our efforts include a redetermination of the radial extent of the starburst cluster and a detailed analysis of its $K$ band luminosity function (Nürnberger \& Petr-Gotzens 2002), a search for mid infrared sources located within and in the vicinity of the cluster (Nürnberger \& Stanke 2002) as well as a thorough characterization of these mid infrared sources in terms of their location and evolutionary stage (Nürnberger 2002a, 2002b; Nürnberger et al. 2002).

\section{The NGC 3603 OB cluster and its $\mathrm{H}$ II region}

NGC 3603 (see Fig. 1) - located in the Carina spiral arm $(l \sim 291.6, b \sim-0.5)$ at a distance of about $7 \mathrm{kpc}$ (e.g.
Moffat 1983; Clayton 1986; Melnick et al. 1989; De Pree et al. 1999) - is one of the most luminous, optically visible H II regions in our galaxy, powered by a massive cluster of OB stars (Goss \& Radhakrishnan 1969). It is frequently compared to the 30 Dor giant $\mathrm{H}$ II region in the Large Magellanic Cloud, although 30 Dor has about 7 times the total ionization flux, is about 10 times larger in diameter, and about 40 times more massive (Balick et al. 1980; Kennicutt 1984; Moffat et al. 1994). Also, the NGC 3603 OB cluster itself is a scaled down galactic version of the R 136 cluster, the primary source of excitation in 30 Dor. It shows the highest density of optically visible high mass stars known in the Galaxy (Melnick et al. 1989; Moffat et al. 1994; Drissen et al. 1995; Hofmann et al. 1995).

Due to its proximity NGC 3603 plays a key role - as galactic starburst template - in the understanding of extragalactic starburst regions. Unlike nearby regions of high mass star formation, such as the well known Orion Nebula, the combined effort of the dense cluster of hot stars has an even more severe impact on the surrounding gas and dust: on the one hand by providing a huge amount of ionizing photons (Lyman continuum flux $\sim 10^{51} \mathrm{~s}^{-1}$; Kennicutt 1984; Drissen et al. 1995) and 
Table 1. Observational parameters.

\begin{tabular}{lccccccc}
\hline \hline Molecule & Transition & $\begin{array}{c}\text { Rest frequency } \\
{[\mathrm{GHz}]}\end{array}$ & $\begin{array}{c}\text { Velocity resolution } \\
{\left[\mathrm{km} \mathrm{s}^{-1}\right]}\end{array}$ & $\begin{array}{c}\text { Beam }(F W H M) \\
{[\operatorname{arcsec}]}\end{array}$ & $\eta_{\mathrm{mb}}$ & $\begin{array}{c}T_{\text {sys }} \\
{[\mathrm{K}]}\end{array}$ & $\begin{array}{c}\tau_{\text {atm }} \\
\text { CS }\end{array}$ \\
& $J=2-1$ & 97.981 & $0.129 \pm 0.002$ & 52.0 & 0.73 & $160 \pm 17$ & $0.20 \pm 0.03$ \\
& $J=3-2$ & 146.969 & $0.086 \pm 0.001$ & 34.0 & 0.60 & $326 \pm 45$ & $0.10 \pm 0.07$ \\
\hline
\end{tabular}

on the other hand by (further) compressing the adjacent molecular cloud through fast stellar winds (up to several $100 \mathrm{~km} \mathrm{~s}^{-1}$; Balick et al. 1980). The energetic Lyman continuum photons and stellar winds evaporate and disperse density enhancements of the interstellar medium, giving rise to protoplanetary-disk(proplyd-) and / or pillar-like phenomena (Brandner et al. 2000; Brandl et al. 1999). Furthermore, stellar winds originating from HD 97950, the Trapezium-like stellar center of the cluster, are considered the driving sources of a bubble which expands with velocities of about $50 \mathrm{~km} \mathrm{~s}^{-1}$ into the ambient medium (Balick et al. 1980; Clayton 1986, 1990).

First evidence of molecular gas and dust associated with NGC 3603 is given by HiRes processed IRAS data (Fig. 2). In particular, the $100 \mu \mathrm{m}$ image shows two unresolved condensations of relatively cold material, while both the $12 \mu \mathrm{m}$ and $25 \mu \mathrm{m}$ data sets apparently trace regions of relatively warm gas and dust close to the $\mathrm{OB}$ cluster. Furthermore, the ring-/shell-like feature seen at $60 \mu \mathrm{m}$ is comparable to the distribution of far infrared emission observed towards the Rosette GMC (see Fig. 2 of Williams et al. 2000 as well as Williams \& Blitz 1998) and might be indicative of a high star formation rate. Although $\mathrm{mm}$ observations are best suited to investigate physical and chemical properties of star forming environments, so far only a small part of the NGC 3603 giant molecular cloud (hereafter abbreviated as NGC 3603 GMC) has been studied at millimeter wavelengths. Of course, NGC 3603 was covered by galactic CO surveys (e.g. Grabelsky et al. 1987, 1988), but ${ }^{12} \mathrm{CO}(J=1-0)$ observations at higher spatial resolution - covering an area of about $6^{\prime} \times 6^{\prime}-$ were needed to reveal the spatial distribution and clumpiness of the molecular gas right towards the south of the OB cluster (Melnick 1989).

Because ${ }^{12} \mathrm{CO}$ is optically thick throughout the GMC, however, Melnick's map does not provide too much information on the gas properties. In order to infer the density and temperature structure, the kinematics as well as the chemical composition of the gas more sensitive (including optically thin) molecular tracers must be taken into account. Therefore we are carrying on a thorough study of the molecular environment of NGC 3603.

\section{Observations and data reduction}

Due to its southern declination $\left(\delta \sim-61^{\circ}\right)$ NGC 3603 is best accessible at millimeter wavelengths with the $15 \mathrm{~m}$ SwedishESO Submillimetre Telescope (SEST). To get insight on the physical parameters and chemical composition our study comprises several transitions of $\mathrm{CO}$ and $\mathrm{CS}$, as well as some of their isotopomers. Here we focus on the data sets of the high density tracers CS $(J=2-1)$ and CS $(J=3-2)$, which were obtained at the SEST during three observing runs in November
1995, January 1997 and January 1998 by using the facility $2.0 \mathrm{~mm} / 3.0 \mathrm{~mm}$ SIS receiver in combination with an acousto optical spectrometer (AOS) in high resolution mode. Sky subtraction was done through dual beam switching with an azimuthal beam throw of $12^{\prime}$. The scale of the receiver temperature was calibrated using the chopper-wheel method. Table 1 summarizes the observing parameters.

Because we aimed at a fully Nyquist-sampled map in the $\mathrm{CS}(2-1)$ line - at $98 \mathrm{GHz}$ the half power beamwidth (HPBW) is about $52^{\prime \prime}-$ a uniform grid spacing of $25^{\prime \prime}$ was chosen. This results in a slight undersampling of simultaneously observed CS (3-2) data (HPBW 34" at $147 \mathrm{GHz}$ ). A total of 388 and 367 map positions were observed in CS (2-1) and CS (3-2), respectively, covering an area of 5'.8 $\times 16$ '7. For both CS lines the integration time was $180 \mathrm{~s}$ per position. The telescope pointing was regularly checked on Orion $\mathrm{SiO}$ or $\mathrm{R} \mathrm{Crt} \mathrm{and} \mathrm{found} \mathrm{to} \mathrm{be}$ better than $3^{\prime \prime}$. For comparison with optical, infrared and radio data the origin of our millimeter maps was chosen to be identical with the Tycho- $2^{1}$ source $8959-1919-1\left(\mathrm{RA}_{\mathrm{J} 2000.0}=\right.$ $11^{\mathrm{h}} 15^{\mathrm{m}} 15^{\mathrm{s}} .15, \mathrm{DEC}_{\mathrm{J} 2000.0}=-61^{\circ} 17^{\prime} 35^{\prime \prime}$. 1$)$. All offsets are relative to this Tycho- 2 position.

Throughout the observations the atmospheric opacity was stable at a level of $\tau_{\text {zenith }} \sim 0.20 \pm 0.03$ at $98 \mathrm{GHz}$ and $\tau_{\text {zenith }} \sim 0.10 \pm 0.07$ at $147 \mathrm{GHz}$. Flux calibration (within an error of $20 \%$ ) was obtained by additionally monitoring Orion $\mathrm{SiO}$ and IRC +10216 . Homogeneous intensity scaling of the whole map was ensured by repeatedly observing the center positions of two molecular cloud clumps (MM 2 and MM4). The processing of the raw data was performed within the IDL software package. First, all spectra were inspected individually and residual baselines were removed by fitting polynomials, as appropriate. Then, intensities were converted to the main beam temperature scale following the SEST users manual, i.e. the measured antenna temperatures were divided by the corresponding main beam efficiency (see Table 1).

\section{Results}

\subsection{Global spatial distribution}

We present for the first time large scale maps of the dense molecular gas associated with NGC 3603 (Fig. 3). Both the velocity integrated CS $(J=2-1)$ map in the left panel and the velocity integrated CS $(J=3-2)$ map in the right panel cover a total area of about 5'.8 $\times 16$ '7. Comparison of these CS maps with the CO map of Melnick (1989) clearly shows that the molecular cloud extends to the south of the OB cluster much more than can be suspected from Melnick's data. Additionally,

1 The TYCHO-2 catalogue (Hog et al. 2000) is accessible via the Centre de Données astronomiques de Strasbourg (CDS). 

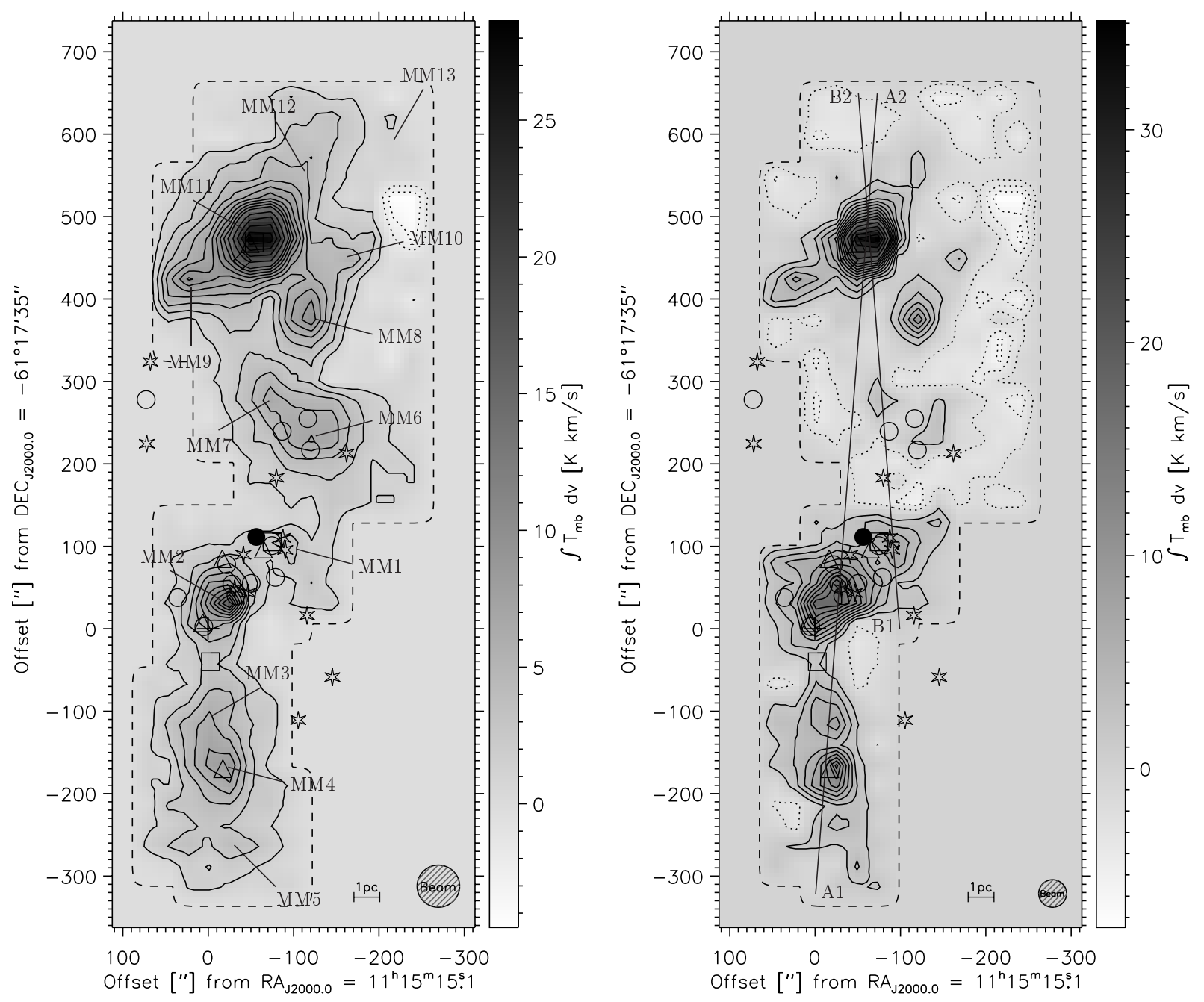

Fig. 3. Total velocity integrated emission observed in the CS (2-1) transition (left) and CS (3-2) transition (right). In both maps the molecular emission is displayed in grey-scale as well as in contours. Solid contour lines indicate positive intensities, while dashed contour lines trace negative intensities (mainly due to noisy spectra, slight contamination by emission in the OFF position, etc.). Contours are drawn at $5 \%$ intervals until the $50 \%$ level, thereafter at $10 \%$ intervals to avoid crowding towards the clump centers. As in Fig. 2 the dot gives the position of the OB cluster, for which we assume the mass-center of the Trapezium-like system HD 97950 (Moffat et al. 1994; Hofmann et al. 1995), and asterisks mark several prominent IR sources (Frogel et al. 1977). Additionally, $\mathrm{OH}, \mathrm{H}_{2} \mathrm{O}$ and $\mathrm{CH}_{3} \mathrm{OH}$ maser sources are indicated by squares, triangles and diamonds, respectively (Braz \& Epchtein 1983; Caswell \& Haynes 1987; Caswell 1998; Caswell et al. 1989; Caswell et al. 1995). Open circles denote maxima of the $3.4 \mathrm{~cm}$ emission, which were identified with the Australia Telescope Compact Array (ATCA) by De Pree et al. (1999). Molecular cloud clumps which are detected in both CS (2-1) and CS (3-2) are labeled in the left panel. In the right panel two straight lines indicate cuts along which position-velocity diagrams are calculated (see Fig. 5 and Sect. 4.2). Note that the same symbols are used throughout this paper.

our data sets reveal several massive clumps of molecular gas in the north of the OB cluster, a region which was partly studied in CS (2-1) by Zinchenko et al. (1995). Because we tried to trace the preference direction of the proposed star formation scenario (see Sect. 5) the maps are slightly biased towards a north-south orientation. However, this does not affect any conclusions in this paper.

Molecular cloud clumps which are detected in both CS (2-1) and CS (3-2) are labeled MM 1 through MM 13 in the left panel of Fig. 3. Note that clump MM 13 is only seen in individual channel maps (see Fig. 4) but does not appear in the velocity integrated maps. The positions of all identified clumps are given in Table 2 together with the line parameters as determined by the best Gaussian fit(s) to the observed line profile, i.e. the velocity $v_{\mathrm{LSR}}^{\text {peak }}$ and the main beam temperature $T_{\mathrm{mb}}^{\text {peak }}$ measured at the peak(s), the full width at half maximum intensity $\Delta v_{F W H M}$ as well as the intensity integrated over the individual Gaussian(s) $\int T_{\mathrm{mb}} \mathrm{d} v$. There is good agreement between the parameters of the CS (2-1) profiles and those of the CS (3-2) lines. Our results for MM 2 and MM 11 agree well with those of Zinchenko et al. (1995).

\subsection{Kinematic structure}

In addition to the spatial distribution of the molecular gas, our CS data provide, as well, valuable new information on the kinematics of the cloud clumps, which is displayed in channel maps 
Table 2. Observed spectral line parameters.

\begin{tabular}{|c|c|c|c|c|c|c|c|c|c|c|}
\hline \multirow[t]{2}{*}{ Clump } & \multicolumn{2}{|c|}{ Offset position $^{a}$} & \multicolumn{4}{|c|}{$\mathrm{CS}(2-1)^{b}$} & \multicolumn{4}{|c|}{$\mathrm{CS}(3-2)^{c}$} \\
\hline & $\begin{array}{c}\Delta \alpha \\
{[\operatorname{arcsec}]}\end{array}$ & $\begin{array}{c}\Delta \delta \\
{[\operatorname{arcsec}]}\end{array}$ & $\begin{array}{c}v_{\mathrm{LSR}}^{\text {peak }} \\
{\left[\mathrm{km} \mathrm{s}^{-1}\right]}\end{array}$ & $\begin{array}{c}T_{\mathrm{mb}}^{\text {peak }} \\
{[\mathrm{K}]} \\
\end{array}$ & $\begin{array}{l}\Delta v_{F W H M} \\
{\left[\mathrm{~km} \mathrm{~s}^{-1}\right]}\end{array}$ & $\begin{array}{c}\int T_{\mathrm{mb}} \mathrm{d} v \\
{\left[\mathrm{~K} \mathrm{~km} \mathrm{~s}^{-1}\right]}\end{array}$ & $\begin{array}{c}v_{\mathrm{LSR}}^{\text {peak }} \\
{\left[\mathrm{km} \mathrm{s}^{-1}\right]}\end{array}$ & $\begin{array}{c}T_{\mathrm{mb}}^{\text {peak }} \\
{[\mathrm{K}]}\end{array}$ & $\begin{array}{l}\Delta v_{F W H M} \\
{\left[\mathrm{~km} \mathrm{~s}^{-1}\right]}\end{array}$ & $\begin{array}{c}\int T_{\mathrm{mb}} \mathrm{d} v \\
{\left[\mathrm{~K} \mathrm{~km} \mathrm{~s}^{-1}\right]}\end{array}$ \\
\hline MM 1 & -90 & +100 & 12.1 & 0.6 & 3.8 & 2.4 & 12.3 & 1.4 & 3.8 & 5.6 \\
\hline \multirow[t]{2}{*}{ MM 2} & -25 & +30 & 13.0 & 1.4 & 3.3 & 4.8 & 12.9 & 2.1 & 3.4 & 7.6 \\
\hline & & & 16.2 & 0.8 & 3.3 & 2.8 & 16.0 & 1.4 & 3.4 & 5.2 \\
\hline MM 3 & 0 & -110 & 15.6 & 0.8 & 4.6 & 3.8 & 15.4 & 0.7 & 4.3 & 3.1 \\
\hline \multirow[t]{2}{*}{ MM 4} & -15 & -165 & 12.9 & 0.3 & 3.3 & 0.9 & 12.9 & 0.4 & 3.3 & 1.3 \\
\hline & & & 16.3 & 1.4 & 3.4 & 4.9 & 16.3 & 1.5 & 3.4 & 5.5 \\
\hline MM 5 & -30 & -260 & 16.0 & 0.7 & 2.0 & 1.5 & 16.0 & 0.7 & 1.6 & 1.2 \\
\hline \multirow[t]{2}{*}{ MM 6} & -120 & +230 & 12.9 & 0.3 & 3.3 & 1.1 & 12.9 & 0.5 & 3.3 & 1.1 \\
\hline & & & 15.3 & 0.3 & 3.3 & 1.8 & - & - & - & - \\
\hline MM 7 & -75 & +280 & 14.0 & 0.6 & 2.9 & 1.7 & 14.0 & 0.7 & 2.3 & 1.7 \\
\hline MM 8 & -120 & +380 & 13.4 & 1.8 & 3.2 & 6.0 & 13.3 & 2.0 & 3.1 & 6.5 \\
\hline \multirow[t]{2}{*}{ MM 9} & +20 & +425 & 12.9 & 0.6 & 4.0 & 2.6 & 12.9 & 0.2 & 4.0 & 0.9 \\
\hline & & & 16.3 & 1.4 & 4.2 & 6.1 & 16.3 & 1.3 & 4.2 & 5.9 \\
\hline \multirow[t]{2}{*}{ MM 10} & -160 & +450 & 13.0 & 0.6 & 3.0 & 1.9 & 13.3 & 0.4 & 3.0 & 1.3 \\
\hline & & & 15.3 & 1.2 & 2.2 & 2.7 & 15.4 & 1.0 & 1.4 & 1.5 \\
\hline MM 11 & -60 & +470 & 13.9 & 3.5 & $6.6^{d}$ & 24.5 & 13.9 & 3.6 & $6.6^{d}$ & 25.5 \\
\hline MM 12 & -110 & +550 & 10.8 & 0.9 & 3.8 & 3.7 & 10.8 & 0.6 & 3.3 & 2.0 \\
\hline MM 13 & -215 & +550 & 15.6 & 1.1 & 2.1 & 2.3 & 15.6 & 0.9 & 1.9 & 1.7 \\
\hline
\end{tabular}

Notes: ${ }^{a}$ the reference position is $\mathrm{RA}_{\mathrm{J} 2000.0}=11^{\mathrm{h}} 15^{\mathrm{m}} 15.1, \mathrm{DEC}_{\mathrm{J} 2000.0}=-61^{\circ} 17^{\prime} 35^{\prime \prime} ;{ }^{b} T_{\mathrm{rms}}^{\mathrm{CS}(2-1)}=0.04 \mathrm{~K}$ on average ${ }^{c} T_{\mathrm{rms}}^{\mathrm{CS}(3-2)}=0.06 \mathrm{~K}$ on average; ${ }^{d}$ very broad line profile, likely due to turbulence.

of the CS (2-1) and CS (3-2) emission (Fig. 4). These maps cover the velocity range from $+6.0 \mathrm{~km} \mathrm{~s}^{-1}$ to $+23.0 \mathrm{~km} \mathrm{~s}^{-1}$ in steps of $1.0 \mathrm{~km} \mathrm{~s}^{-1}$. As in the total velocity integrated maps, presented in Fig. 3, contour lines are drawn at 5\% intervals until the $50 \%$ level, thereafter at $10 \%$ intervals. The position of the OB cluster is given by a dot in all panels, while the IR sources, masers and $3.4 \mathrm{~cm}$ maxima (markers are the same as in Fig. 3) are indicated only in the first panel of each row. Because the brightest clump with the broadest line profiles, MM 11, is less dominant than for the total velocity integrated maps, the channels maps provide a better intensity contrast for weak sources with narrow line profiles. Therefore, tracing the CS emission channel-by-channel allows to identify weak structures like MM 12 and MM 13 and to separate the individual clumps like MM 8 and MM 10.

We also present position-velocity diagrams (Fig. 5) along two cuts through the cube of the CS (3-2) data. These cuts are indicated in the right panel of Fig. 3. The velocities measured at the loci of the peak emission are typically in the range from $v_{\mathrm{LSR}} \sim 11 \mathrm{~km} \mathrm{~s}^{-1}$ to $v_{\mathrm{LSR}} \sim 16 \mathrm{~km} \mathrm{~s}^{-1}$ (see also Table 2). Hence, by calculating the mean value the systematic velocity of NGC 3603 is $v_{\mathrm{LSR}} \sim 14.2 \pm 1.6 \mathrm{~km} \mathrm{~s}^{-1}$.

Assuming that NGC 3603 closely follows the galactic differential rotation and taking into account a proper correction for the peculiar radial velocity (Stark \& Brand 1989; Fich et al. 1989), a systematic velocity of $v_{\mathrm{LSR}} \sim 14.2 \pm 1.6 \mathrm{~km} \mathrm{~s}^{-1}$ implies a kinematic distance of $7.7 \pm 0.2 \mathrm{kpc}$. This value is in excellent agreement with that recently reported by Crowther \& Dessart $(1998 ; 7.9 \mathrm{kpc})$ based on spectroscopic and photometric data of individual cluster stars together with $E(B-V)=1.40$. Other estimates are from Goss et al. (1972; see also Goss \& Radhakrishnan 1969; 8.4 kpc), Moffat (1974; $8.1 \pm 0.8 \mathrm{kpc})$, van den Bergh (1978; 7.2 $\left.{ }_{-1.0}^{+0.8} \mathrm{kpc}\right)$, Melnick et al. (1989; $7.2 \mathrm{kpc})$, Moffat $(1983 ; 7.0 \mathrm{kpc})$, Pandey et al. (2000; $6.3 \pm 0.6 \mathrm{kpc})$, De Pree et al. (1999; $6.1 \pm 0.6 \mathrm{kpc})$ and Shobbrook \& Lynga (1994; $5.2 \mathrm{kpc})$.

Both the channel maps (Fig. 4) and the position-velocity diagrams (Fig. 5) reveal the remarkably broad width of the line profiles towards clump MM 11 (see also the corresponding CS spectra in Fig. 6). These lines of widths $6.6 \mathrm{~km} \mathrm{~s}^{-1}$ suggest that the molecular material in the clump is highly turbulent.

\subsection{Spectral signatures}

In Fig. 6 we present the CS spectra as observed towards the central line-of-sight of each clump. Their positions might be identified with the help of the accompanying CS (2-1) map of the cloud. Most of the line profiles can be easily fit by a single Gaussian which suggests a rather uniform velocity structure for the corresponding clumps (except for MM 11 as discussed in Sects. 4.2 and 5.1.3). Although the interpretation of our data sets is limited by the low spatial resolution, the line profiles of five clumps (MM2, MM4, MM 6, MM9 and MM 10) are clearly composed of two components, which were fitted by individual Gaussians.

While in all cases the blue component is centered at $v_{\mathrm{LSR}} \sim 13 \mathrm{~km} \mathrm{~s}^{-1}$, the red component is found at $v_{\mathrm{LSR}}=15.3-16.3 \mathrm{~km} \mathrm{~s}^{-1}$, respectively. Such separations 

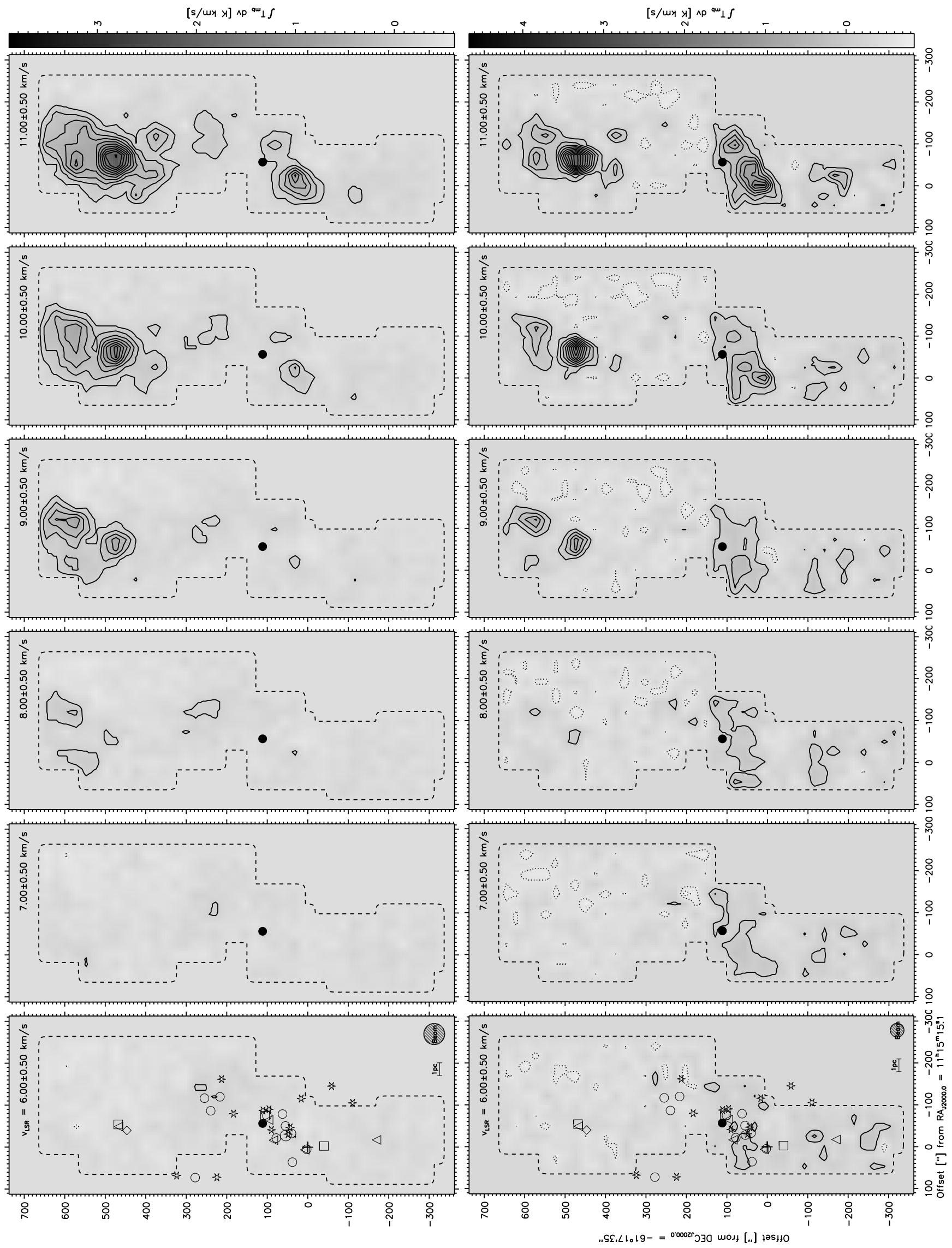

Fig. 4. Channel maps of the CS (2-1) emission (left) and of the CS (3-2) emission (right). Symbols are the same as in Fig. 3. 

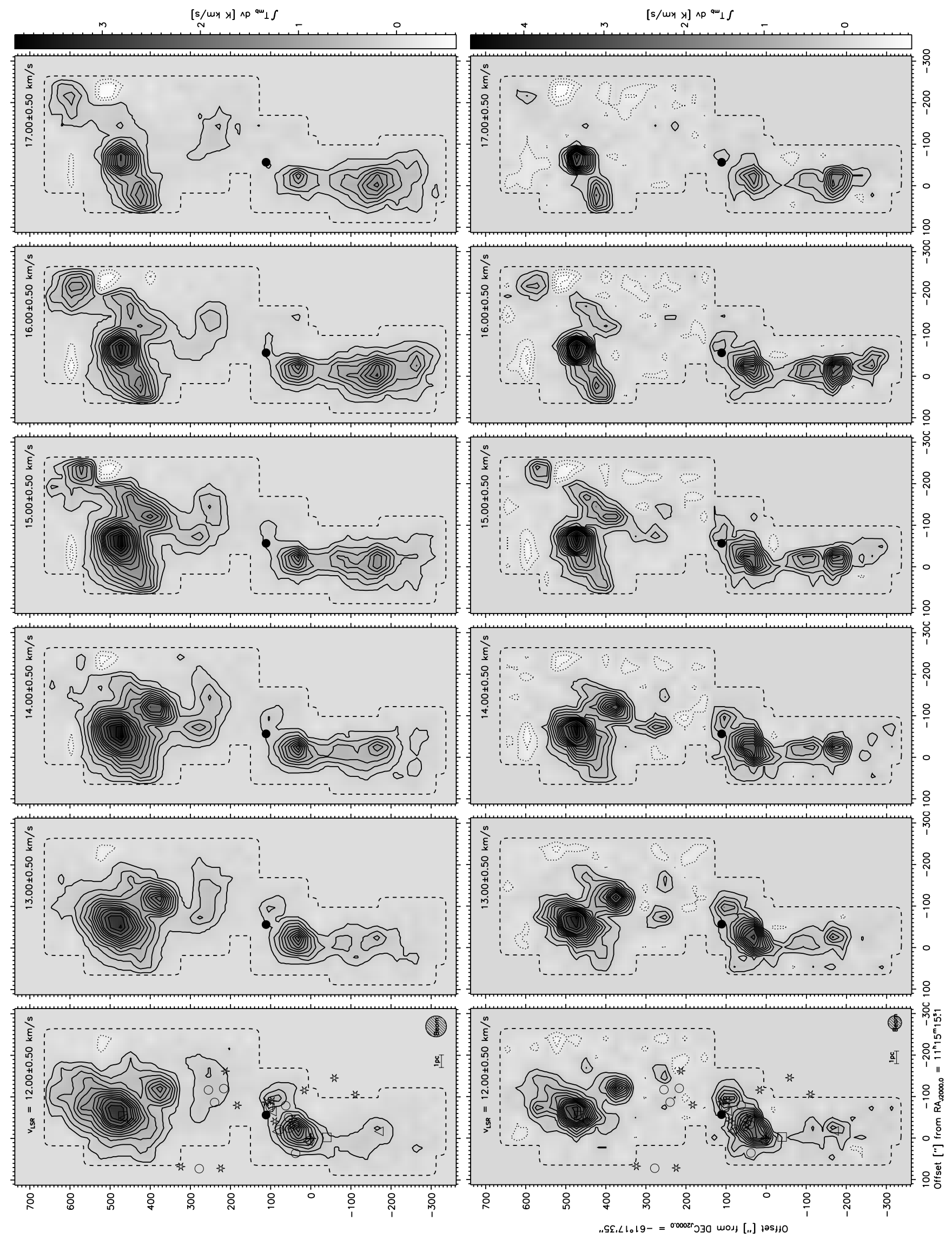

Fig. 4. continued. 


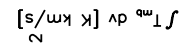
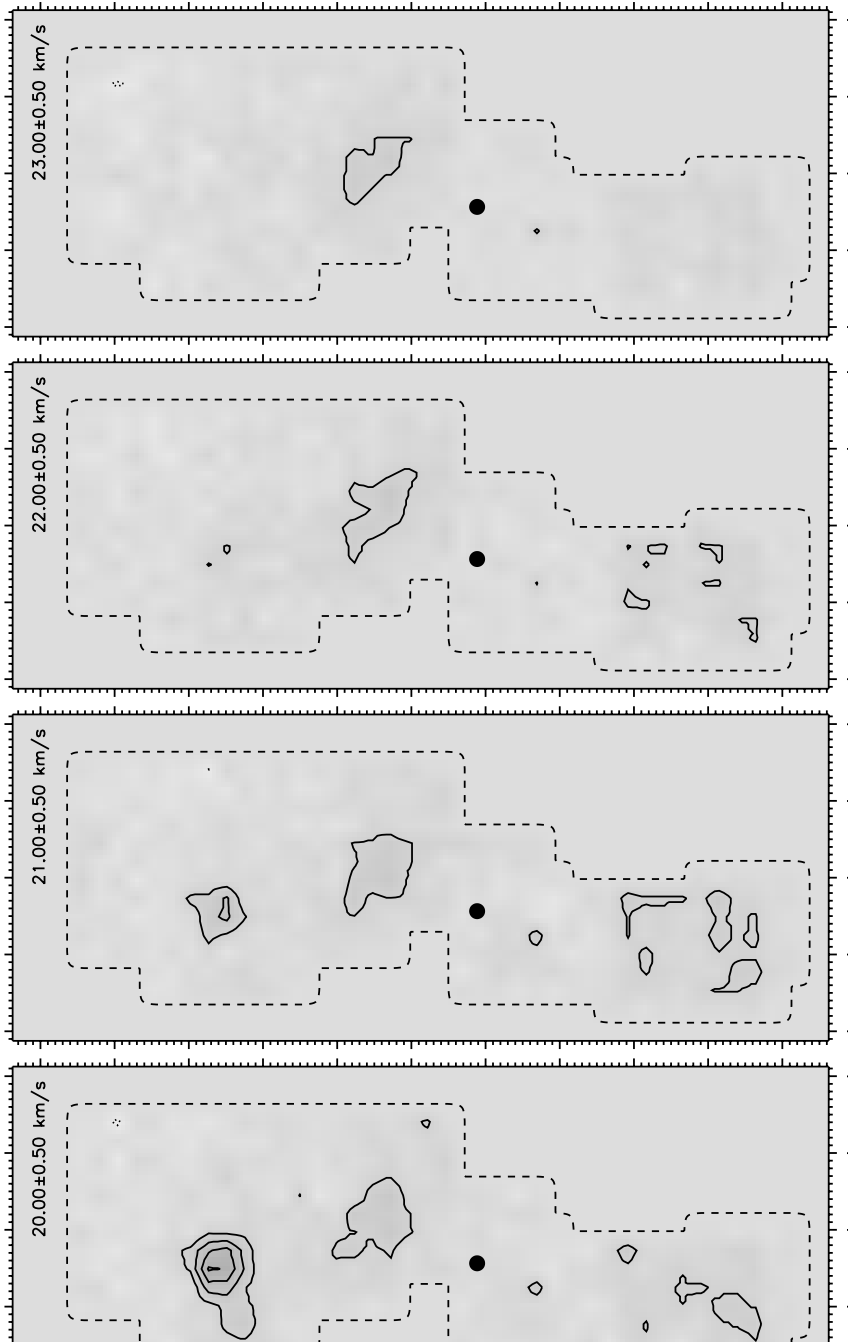

(घ)
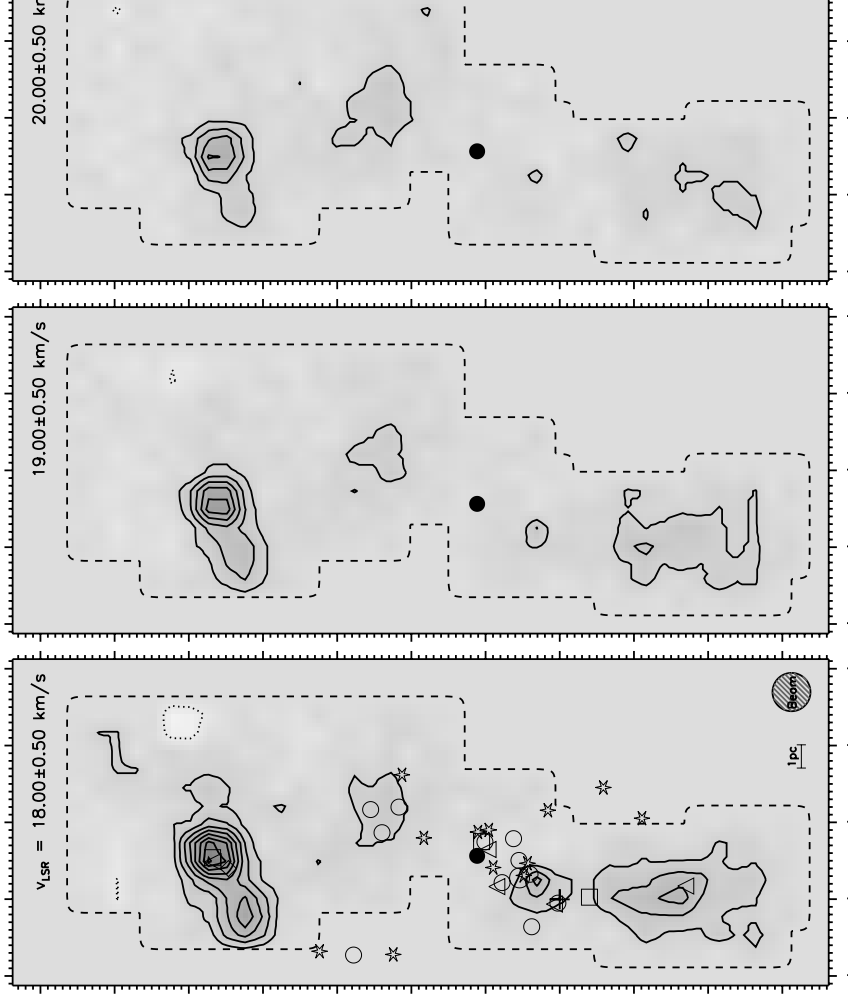

๖ $[s / W x$ y y $] \wedge \rho^{a w} \perp S$
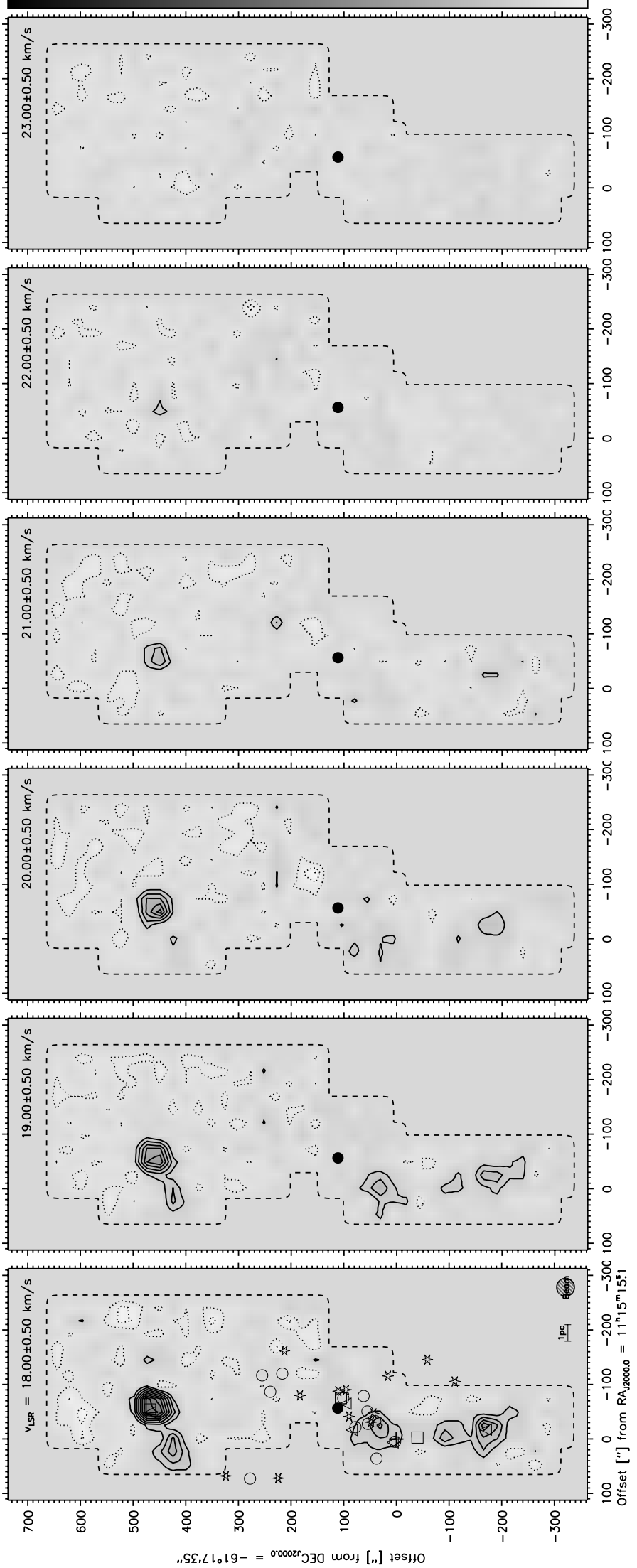

Fig. 4. continued. 

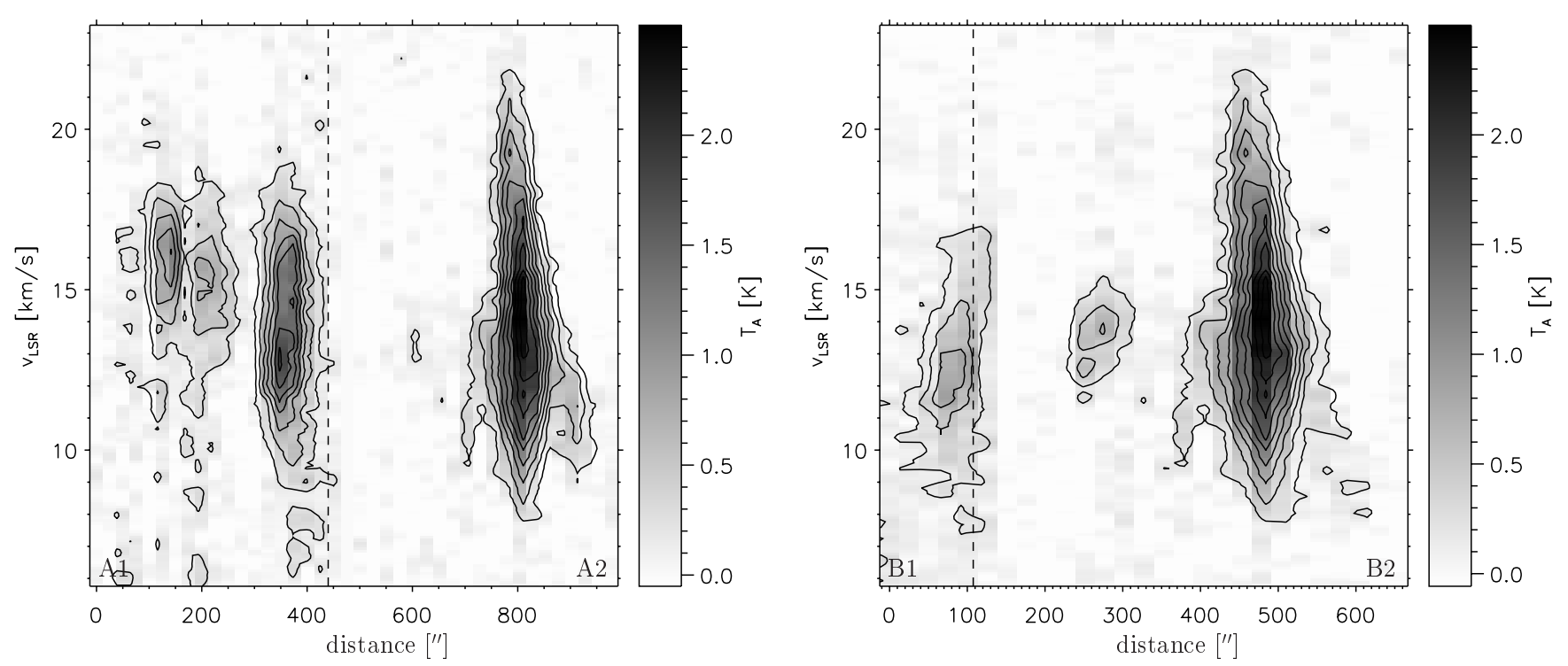

Fig. 5. Position-velocity diagrams along two cuts through the CS (3-2) data cube as indicated in Fig. 3. For each cut the position closest to the OB cluster is given by a dashed vertical line.

by about $3 \mathrm{~km} \mathrm{~s}^{-1}$ would convert into distance differences of about $300 \mathrm{pc}$ along the line-of-sight, when we only take into account the distances and systematic velocities of NGC 3603 ( $D \sim 7 \mathrm{kpc}, v_{\mathrm{LSR}} \sim 14 \mathrm{~km} \mathrm{~s}^{-1}$ ) and the "neighbouring" (in projection) $\mathrm{H}$ II region NGC $3576\left(D \sim 3 \mathrm{kpc}, v_{\mathrm{LSR}} \sim-23 \mathrm{~km} \mathrm{~s}^{-1}\right.$; De Pree et al. 1999). However, as both clump components most likely are subject to complex kinematics (see e.g. De Pree et al. 1999; Georgelin et al. 2000) within the NGC 3603 H II region, much smaller effective line-of-sight separations seem to be more appropriate.

\subsection{Clump radii}

The clump sizes are determined through the area where the velocity integrated line intensity exceeds one half of its maximum value. For that purpose we used the CS (3-2) data which provide a slightly better spatial resolution than the CS (2-1) data, and calculated the average projected radius from $6-8$ cuts through each clump. At first sight, these values (see Table 3) are typically of the order $0.8 \pm 0.2 \mathrm{pc}$, assuming a distance of $7 \mathrm{kpc}$.

However, considering the spatial sampling (i.e. the small number of pixels within the telescope beam) and the observational errors (due to, e.g., noise, pointing inaccuracies and insufficient baseline subtraction), for most clumps the derived diameters resemble closely the FWHM size of the beam (34", corresponding to $\sim 1.2 \mathrm{pc}$ ). The rather roundish clump shapes, in particular those of the northern clumps MM 8 to MM 13, are suspicious in the same way. Hence, the true clump sizes may be considerably smaller (by an unknown factor) than those given in Table 3. The only exceptions are clump MM 2 - its average radius is confirmed by $\mathrm{C}^{18} \mathrm{O}(2-1)$ data with higher spatial resolution (see Fig. 8) - and probably clumps MM 6 and MM 7.
Table 3. Estimates of the clump radii, virial masses and column densities based on the CS (3-2) data.

\begin{tabular}{cccc}
\hline \hline Clump & $\begin{array}{c}\mathcal{R}^{a} \\
{[\mathrm{pc}]}\end{array}$ & $\begin{array}{c}\mathcal{M}_{\mathrm{vir}}^{n=2} \\
{\left[10^{3} \mathcal{M}_{\odot}\right]}\end{array}$ & $\begin{array}{c}\mathcal{N}\left(\mathrm{H}_{2}\right)^{n=2} \\
{\left[10^{23} \mathrm{~cm}^{-2}\right]}\end{array}$ \\
\hline \hline MM 1 & $<0.4^{b}$ & $<0.7$ & $>1.0$ \\
MM2 & $1.0^{c}$ & 1.5 & 0.4 \\
& & 1.5 & 0.4 \\
MM3 & $<0.8$ & $<1.9$ & $>0.7$ \\
MM 4 & $<0.7$ & $<1.0$ & $>0.5$ \\
& & $<1.0$ & $>0.5$ \\
MM 5 & $<0.8$ & $<0.3$ & $>0.1$ \\
MM 6 & $0.9^{d}$ & 1.2 & 0.3 \\
MM7 & $0.9^{d}$ & 0.6 & 0.2 \\
MM 8 & $<0.6$ & $<0.7$ & $>0.5$ \\
MM9 & $<0.9$ & $<1.8$ & $>0.5$ \\
& & $<2.0$ & $>0.6$ \\
MM 10 & $<0.6$ & $<0.7$ & $>0.5$ \\
& & $<0.1$ & $>0.1$ \\
MM 11 & $<0.7$ & $<3.8$ & $>1.8$ \\
MM 12 & $<0.6$ & $<0.8$ & $>0.5$ \\
MM 13 & $<0.5^{e}$ & $<0.2$ & $>0.2$ \\
\hline
\end{tabular}

Notes: ${ }^{a}$ unless otherwise noted, radii $\mathcal{R}$ are derived from the CS (3-2) data and represent upper limits (see Sect. 4.4 for details); ${ }^{b}$ upper limit of radius $\mathcal{R}$ is derived from the $\mathrm{C}^{18} \mathrm{O}(2-1)$ data (Fig. 8); ${ }^{c}$ radius $\mathcal{R}$ derived from the CS data is confirmed by the $\mathrm{C}^{18} \mathrm{O}(2-1)$ data (Fig. 8; please note that we discuss the whole clump MM 2 and not the individual substructures MM $2 \mathrm{~A}$ to MM $2 \mathrm{~F}) ;{ }^{d}$ for both MM 6 and MM 7 the radii $\mathcal{R}$ derived from the CS (3-2) data appear to be reasonable estimates, although no data with higher angular resolution are available for confirmation; ${ }^{e}$ estimated from the channel maps in Fig. 4. 

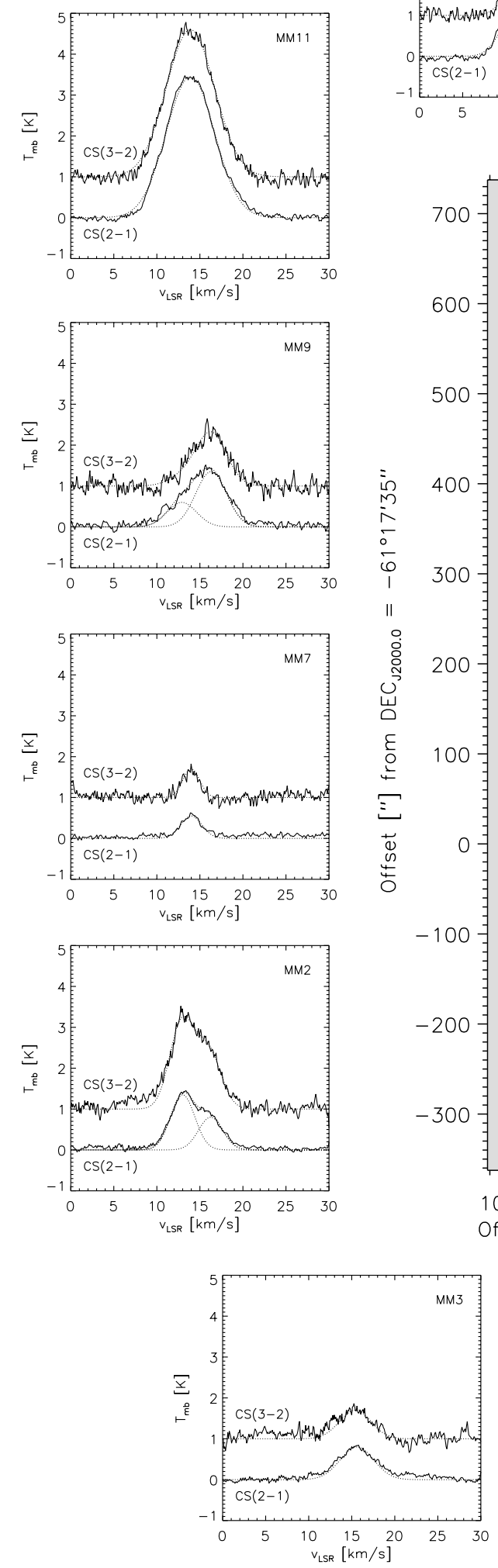
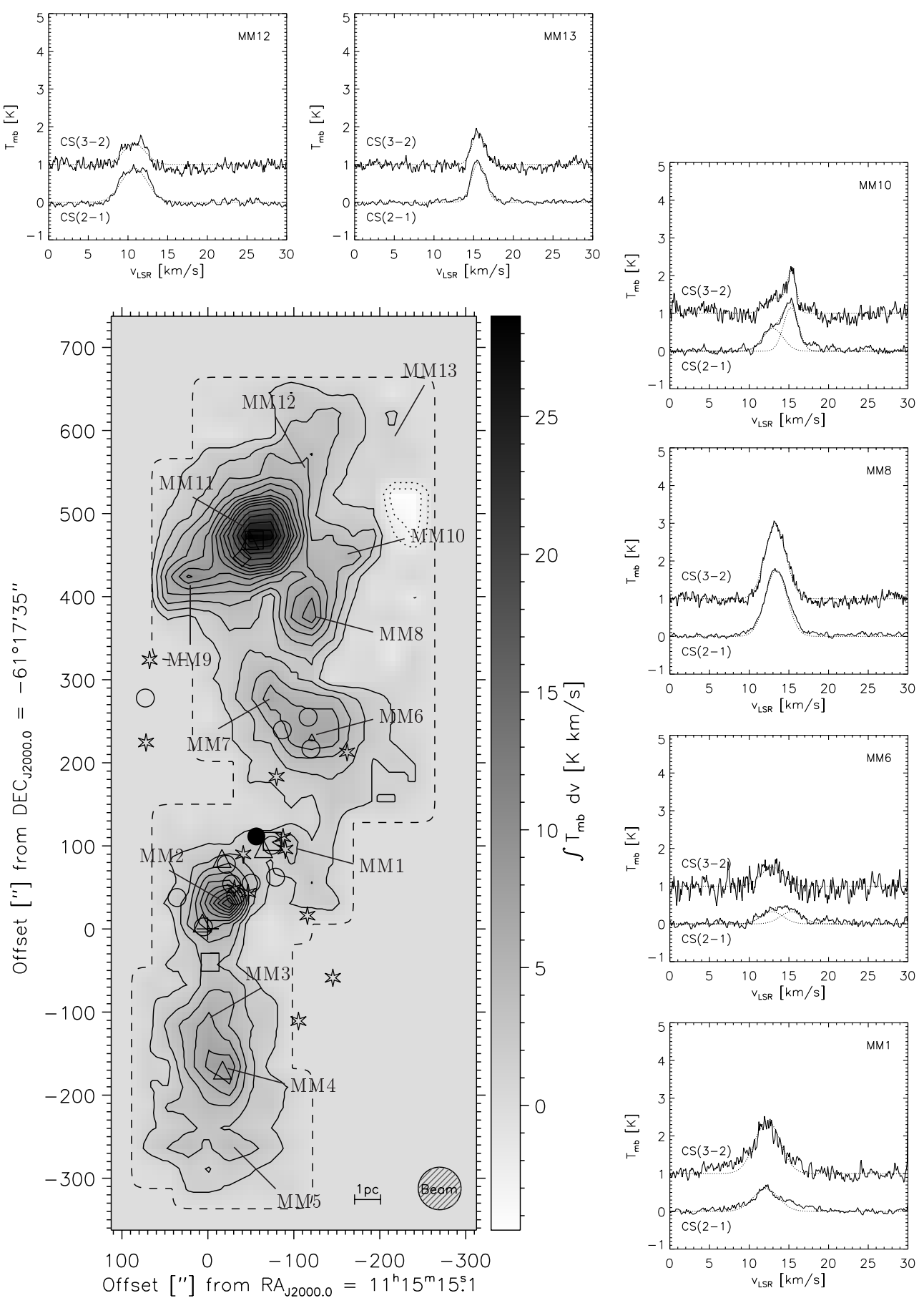
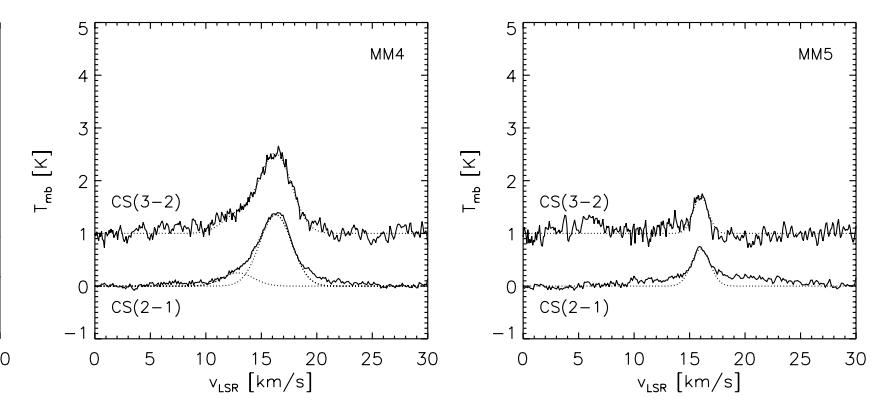

Fig. 6. CS spectra as observed towards the center of all identified molecular cloud clumps. In order to ease the orientation and identification, the spectra are arranged around the velocity integrated CS (2-1) map which is already shown in Fig. 3. Also note that all CS (3-2) spectra are offset in intensity scale by $1 \mathrm{~K}$. 

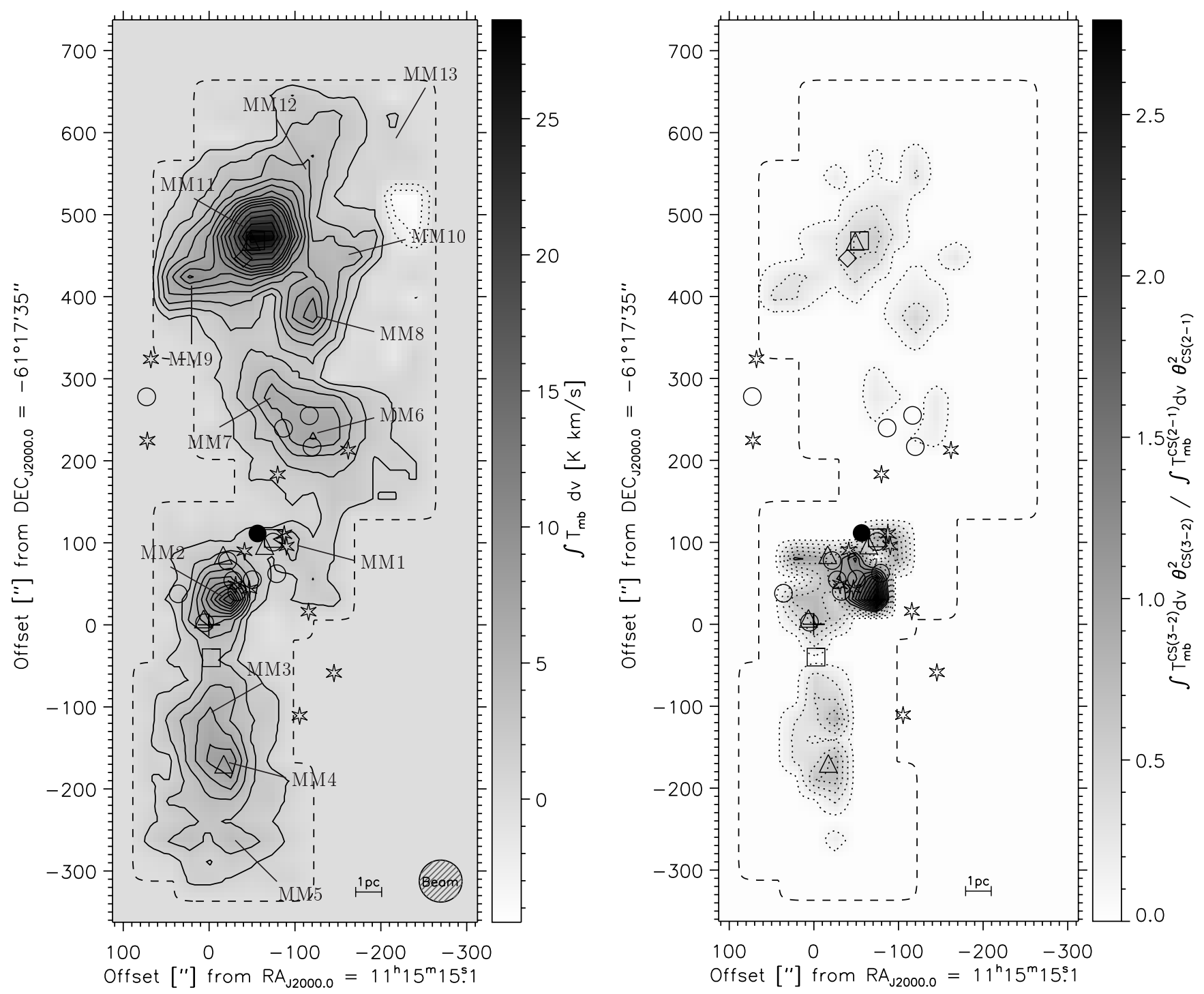

Fig. 7. Intensity ratio of the velocity integrated CS (3-2) and CS (2-1) emission (right panel) in comparison to the CS (2-1) map already known from Fig. 3. Solid contour lines trace intensity ratios larger than unity (from 1.00 to 2.62 in steps of 0.18 ), while ratios lower than unity are indicated by dashed contours (at the levels of $0.10,0.28,0.46,0.64$ and 0.82 ). All other markers are the same as in previous figures.

\subsection{Physical properties}

To get a first idea of the physical conditions within the NGC 3603 molecular cloud we have convolved the CS (3-2) map to the resolution of the CS(2-1) map and then calculated the ratio of the velocity integrated CS maps, i.e. $\left(\int T_{\mathrm{mb}}^{\mathrm{CS}(3-2)} \mathrm{d} v \cdot \theta_{\mathrm{CS}(3-2)}^{2}\right) /\left(\int T_{\mathrm{mb}}^{\mathrm{CS}(2-1)} \mathrm{d} v \cdot \theta_{\mathrm{CS}(2-1)}^{2}\right)$. Almost all positions close to the edges of the mapped area showed low signal-to-noise ratios (close to the average 1 sigma noise level) either in the CS (2-1) or the CS (3-2) line, thus causing artificial maxima or minima in the ratio map; these positions were omitted. We emphasize that the resulting map (Fig. 7) shows lower limits because no clump sizes are considered in the above formula. Taking into account that most of the clumps are probably smaller than the beam $F W H M$ of the CS (3-2) data (Sect. 4.4), we can also constrain upper limits for the CS (3-2)/CS (2-1) ratios which are only moderately larger (by a factor 1.4) than the lower limits.
The CS (3-2)/CS (2-1) ratios displayed in Fig. 7 suggest that both lines are mostly optically thick, if one assumes identical beam filling and high excitation $\left(T_{\mathrm{ex}}^{21}=T_{\mathrm{ex}}^{32}\right)$. However, the molecular gas in the south of the OB cluster has a different characteristic than the gas in the north, i.e. the intensity ratios for MM 1 to MM 4 are clearly higher than those for MM 6 to MM $12(0.80 \pm 0.08$ compared to $0.32 \pm 0.11)$. This indicates either opacities significantly higher for the northern clumps than for the southern ones, or different excitation conditions. The same conclusion would hold if one takes into account clump sizes in the above formula.

Furthermore, one may notice the unusually high CS (3-2) to CS (2-1) ratios (up to 2.8) to the south of the OB cluster and just to the west of clump MM2. These values are consistent with emission from optically thin material, for which one would expect values of $3^{2} / 2^{2}=2.25$. The location coincides with the tip of a spur-like shaped area rather void of molecular emission, which can be identified on optical and near infrared 


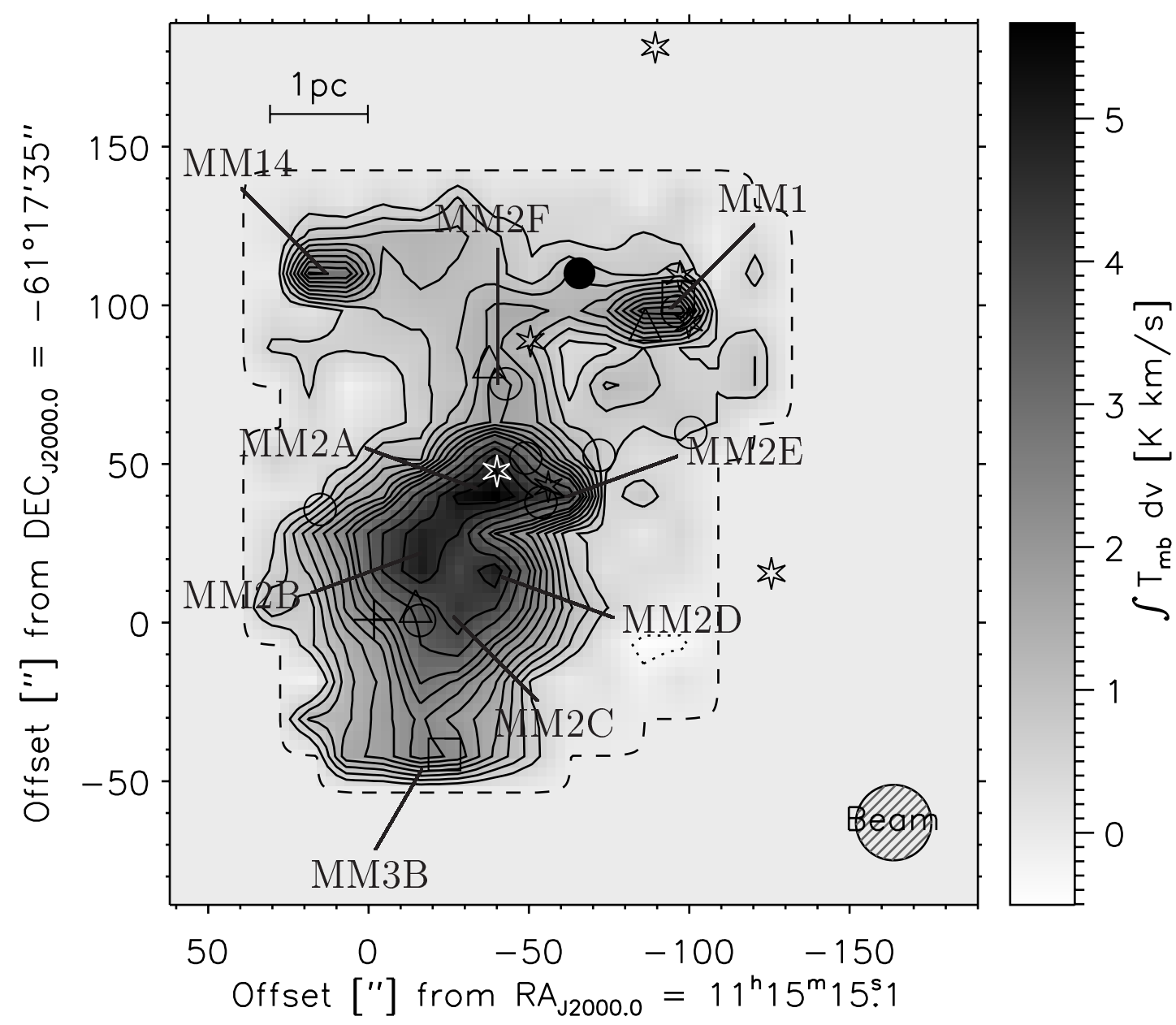

Fig. 8. Substructure of the molecular clump MM 2 as revealed by our $\mathrm{C}^{18} \mathrm{O}(2-1)$ data (beam $F W H M$ is 24 "' 4; Nürnberger 2002b). Individual molecular cloud cores are identified and labeled. All other markers are the same as in previous figures. This data set confirms the clump size of MM 2 derived from the CS (2-1) and CS (3-2) data, but it also shows that the size of clump MM 1 (i.e. the head of the western pillar) is overestimated by the CS data (even the $\mathrm{C}^{18} \mathrm{O}(2-1)$ data may just give an upper limit).

images, too (e.g. Fig. 9). In addition, broadband mid infrared data (Nürnberger \& Stanke 2002) reveal a rim of highly excited gas and dust, which might indicate a shock front originating from the OB cluster and heading for the south/south-west.

The physical properties of the molecular gas located immediately towards the south of the NGC 3603 OB cluster (see Fig. 8) will be investigated in more detail in a forthcoming paper. Then, we will also take into account our CS (5-4), ${ }^{13} \mathrm{CS}(2-1), \mathrm{C}^{34} \mathrm{~S}(2-1)$ and $\mathrm{C}^{18} \mathrm{O}(2-1)$ data for a more sophisticated LTE modeling.

\subsection{Clump mass and column density via virial theorem}

Following MacLaren et al. (1988) we have estimated virial masses for all 13 molecular clumps. Assuming a simple spherical geometry and a Gaussian velocity distribution - i.e. neglecting magnetic fields and internal energy sources - the total mass of a clump can be calculated from the clump radius $\mathcal{R}$ and the observed line width $\triangle v_{F W H M}$ via the equation

$\mathcal{M}_{\mathrm{vir}}=k \cdot \mathcal{R} \cdot \Delta v_{F W H M}^{2}$, where $\mathcal{M}_{\text {vir }}$ is in units of $\mathcal{M}_{\odot}, \mathcal{R}$ in parsecs and $\Delta v_{F W H M}$ in $\mathrm{km} \mathrm{s}^{-1}$. The value of the constant $k$ strongly depends on the slope of the local density distribution $\rho(r) \propto r^{-n}$ :

$k=\left\{\begin{array}{l}210, \text { for } n=0 \\ 190, \text { for } n=1 \\ 126, \text { for } n=2 .\end{array}\right.$

For the calculation of the virial masses we used the radii given in Table 3. We emphasize that these clump sizes - with the exception of MM 2, MM 6 and MM 7 - may be overestimated by an unknown factor (Sect. 4.4), and the same might also be true for the virial mass estimates because they scale proportional to the clump radii. Additionally, we assumed $n=2$ (i.e. isothermal clumps) which is a good description of density profiles found for a wide range of molecular clumps, either pressurebound or gravitationally bound or starforming (Williams et al. 1995; Williams et al. 2000). For $n=1$ and $n=0$ the virial masses would scale as $\frac{190}{126}$ and $\frac{210}{126}$, respectively. The virial mass estimates have then been used to calculate lower limits of the $\mathrm{H}_{2}$ column densities (Table 3, Col. 4), following the formula

$\mathcal{N}\left(\mathrm{H}_{2}\right)=2.3 \times 10^{19} \cdot \frac{\mathcal{M}_{\mathrm{vir}}}{\mathcal{R}^{2}}$, 


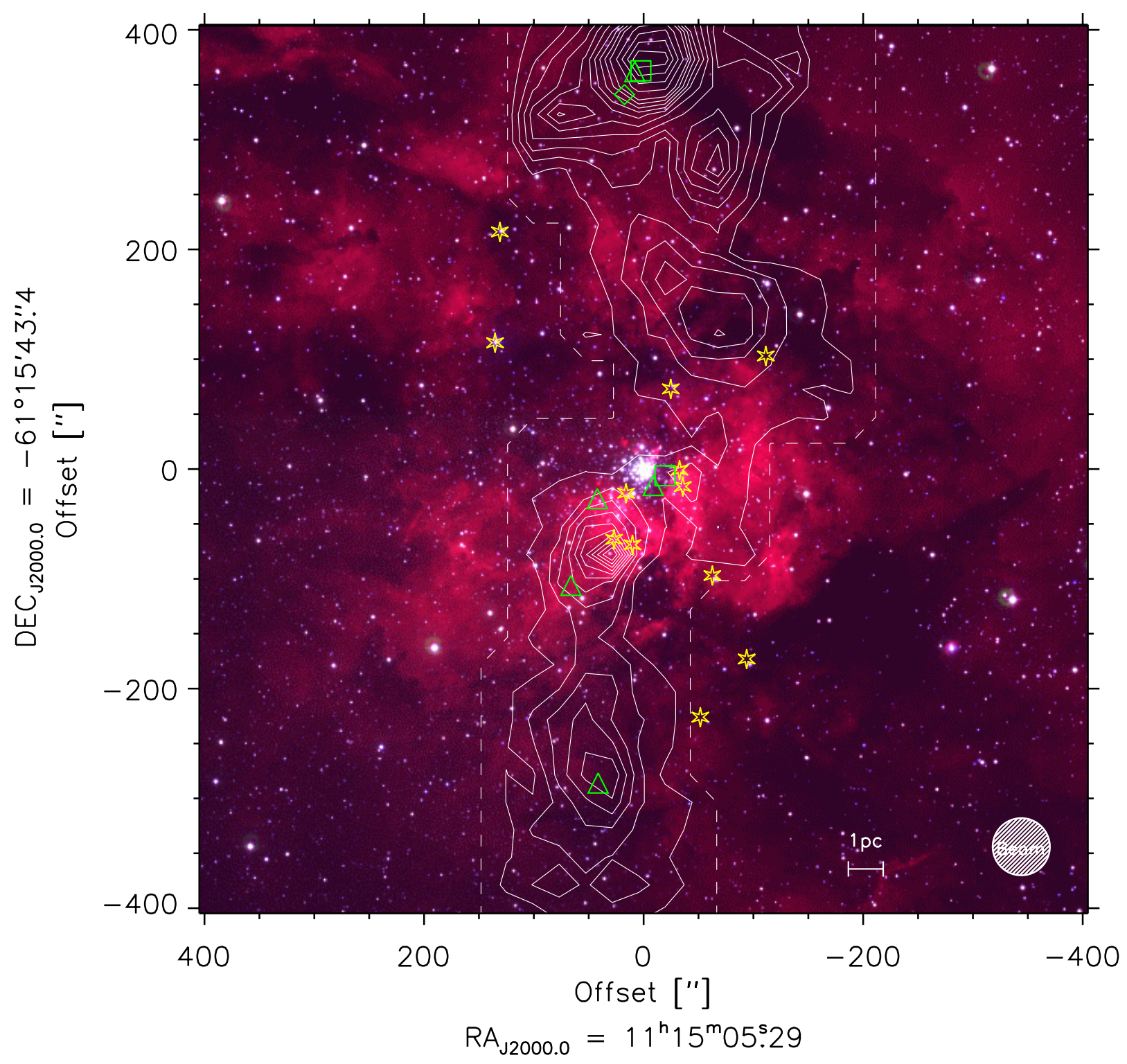

Fig. 9. Zoom-in of the central quarter of the WFI image (Fig. 1) with contour lines of the total velocity integrated CS (2-1) emission overlayed. Markers are as in Fig. 3.

given by Wilson et al. (1999), where again $\mathcal{R}$ is expressed in parsecs.

We find that clump MM11 probably has the highest clump mass and the highest $\mathrm{H}_{2}$ column density: $\mathcal{M}_{\text {vir }}>3.8 \times 10^{3} \mathcal{M}_{\odot}$ and $\mathcal{N}\left(\mathrm{H}_{2}\right)<1.8 \times 10^{23} \mathrm{~cm}^{-2}$ (both derived from the CS (3-2) line, assuming $\rho \propto r^{-2}$ ). These values are about 4 times higher than the corresponding averages of the other clumps: $\left\langle\mathcal{M}_{\text {vir }}\right\rangle \lesssim(1.0 \pm 0.6) \times 10^{3} \mathcal{M}_{\odot}$ and $\left\langle\mathcal{N}\left(\mathrm{H}_{2}\right)\right\rangle \gtrsim(0.4 \pm 0.2) \times 10^{23} \mathrm{~cm}^{-2}$.

Although the NGC 3603 GMC is exposed to high external pressure of energetic winds and ionizing photons, we consider the above virial estimates to be reliable for the following reasons.

First, CS is a tracer of dense molecular gas and, as such, much less (than e.g. CO) affected by the violent environment. Eventually accretion processes might be triggered within the clumps. Motions of infalling gas primarily shift the peak velocities of optically thick lines relative to those of optically thin lines, which - in combination with self-absorption - usually results in double-peaked profiles for optically thick lines (or in an apparent broadening of the optically thick line if the spectral resolution is insufficient; Myers et al. 1996). However, these velocity shifts are only moderate and typically smaller than $0.3 \mathrm{~km} \mathrm{~s}^{-1}$ (e.g. Mardones et al. 1997; Lee et al. 1999; Williams \& Myers 1999).

Second, given the derived virial masses of the order $10^{3} \mathcal{M}_{\odot}$, a relatively large number of young (massive) stars inside the clumps would be needed to make a significant impact on the energy balance of the clumps. But near and mid IR data reveal barely any highly reddened objects, which are deeply embedded within the clumps (Nürnberger \& Stanke 2002). A few reddened sources appear to be located in the neighbourhood of the clumps, possibly blown free of most of the parental molecular gas by stellar winds. 
Third, for clump MM2 we also have CS(5-4) and $\mathrm{C}^{34} \mathrm{~S}(2-1)$ data at hand, which - together with the $\mathrm{CS}(2-1)$ and $\mathrm{CS}(3-2)$ data presented here - allow to estimate the column density independently, e.g. via the CS rotational diagram (Nürnberger 2002b). The result, $\mathcal{N}\left(\mathrm{H}_{2}\right)_{\mathrm{MM} 2}=(0.4 \pm 0.2) \times$ $10^{23} \mathrm{~cm}^{-2}$, is consistent with the one given in Table 3 .

\section{Discussion}

\subsection{Notes on individual clumps}

\subsubsection{1}

Despite of slight offsets - mainly due to the coarse grid spacing of our CS observations - clump MM 1 seems to be consistent with the head of the western pillar seen on optical HST images (Brandner et al. 2000) as well as on near infrared VLT images (Brandl et al. 1999). Apart from its head we did not detected any dense molecular material along the ionized tail of the pillar.

Therefore, judging from our CS data the "elephant trunk" like appearance is caused either by optically thin material which remains undetected in the large SEST beam or by material which is photoevaporated from the dense molecular clump and then blown away from the head of the pillar by stellar winds (pretending the existence of a massive tail on optical and near infrared images). Both scenarios would be in contrast to the famous pillars in M 16, for which Pound (1998) has detected clumpy molecular emission also along the tails.

We also note that the position-velocity diagram along cut B (see the right panel of Fig. 5) indicates for MM 1 a noticable velocity drift with distance from the OB cluster (the closest distance is indicated by the dashed vertical line). Following Pound (1998) such velocity shifts will be obtained if the pillar is located in front of the cluster stars, i.e. if the photoevaporated material is blown towards the observer. However, millimeter interferometric observations are needed to discuss the pillars of NGC 3603 in such detail as those of M 16.

\subsection{2. $\mathrm{MM} 2$}

Clump MM 2 is located $2.5 \mathrm{pc}$ towards the south-east of the NGC 3603 cluster and is hence highly affected by the strong stellar winds and ionizing radiation from the $\mathrm{O}, \mathrm{B}$ and WR type stars. As a consequence of this external influence the clusterfacing side of MM 2 appears to be consistent with the edge of the wind-driven cavity mentioned by Balick et al. (1980) and Clayton (1986, 1990). Thus, both the high radiation and wind pressure of the OB stars may trigger the compression of the molecular gas. Indeed, comparison with $3.4 \mathrm{~cm}$ data of De Pree et al. (1999) shows the rim of MM 2 nicely outlined by photoionized material (Nürnberger et al. 2002).

When overplotting the CS data on an optical image (see Fig. 9) clump MM 2 is located at the bottom of the eastern pillar. In fact, it appears that this pillar protrudes from MM 2, pointing towards the $\mathrm{OB}$ cluster, which is confirmed by our $\mathrm{C}^{18} \mathrm{O}(2-1)$ data (note the substructure MM $2 \mathrm{~F}$ in Fig. 8). Directly at the bottom of the eastern pillar, on the OB cluster facing side of MM2, a sparse association of infrared sources (IRS 9; marked by the white asterisk in Fig. 8) with massive circumstellar shells is found, giving the impression that they were recently revealed from most of their natal environment by stellar winds and ionizing photons (Nürnberger 2002a; Nürnberger et al. 2002).

\subsubsection{11}

Clump MM 11 is situated more than $11 \mathrm{pc}$ to the north of the OB cluster, thus one would not expect too much influence by stellar winds and/or ionizing photons from the massive stars of the cluster. However, on the basis of the very broad line profiles seen in our CS data MM 11 must contain quite a lot of molecular gas and turbulent energy. We derive a virial mass $<3.8 \times 10^{3} \mathcal{M}_{\odot}$ and a $\mathrm{H}_{2}$ column density $>1.8 \times 10^{23} \mathrm{~cm}^{-2}$, assuming a clump radius $<0.7 \mathrm{pc}$. As MM 11 is also associated with all kinds of maser sources $\left(\mathrm{H}_{2} \mathrm{O}, \mathrm{OH}\right.$ and in particular important $\mathrm{CH}_{3} \mathrm{OH}$ ) it appears likely that massive star formation is ongoing in this clump. In fact, $11.9 \mu \mathrm{m}$ and $18 \mu \mathrm{m}$ data recently obtained with TIMMI 2 reveal at least three point sources and three knot-like emission features (Nürnberger \& Stanke 2002).

\subsection{Star formation history of NGC 3603}

Due to the relatively low visual extinction of only 4-5 mag towards the central line-of-sight (Moffat 1983; Melnick et al. 1989) we have the unique opportunity to study the stellar content of the NGC 3603 OB cluster in great detail by photometry and spectroscopy in the optical and infrared. Thus it is well known that the Trapezium-like system HD 97950 in the very core of the cluster contains 3 luminous hydrogen-rich WNL stars, $6 \mathrm{O} 3$ stars, and many other late $\mathrm{O}$ type stars at ages of 2-3 Myr (Melnick et al. 1989; Hofmann et al. 1995). Based on currently available pre-main sequence evolutionary tracks and isochrones (see e.g. D'Antona \& Mazzitelli 1994; Palla \& Stahler 1993, 1999) the majority of intermediate and low mass stars in NGC 3603 appears to be coeval or (probably) slightly younger ( $\$ 1$ Myr; Eisenhauer et al. 1998; Brandl et al. 1999).

On the other hand the presence of several blue supergiants (e.g. Sher 18 and Sher 23; Brandner et al. 1997a) as well as of the potential SN candidate Sher 25 (Brandner et al. 1997a, 1997b), which are associated with but not coeval to the relatively young cluster population, is evidence for a long lasting, continuous process of star formation in NGC 3603. It may have begun 10-20 Myr ago in the north of the OB cluster (Walborn 1973; Melnick et al. 1989) and seems to propagate towards the south, where several reddened sources are found (Frogel et al. 1977; Tapia 1981; Persi et al. 1985; Roth et al. 1987). The presence of a large number of maser sources - including $\mathrm{OH}, \mathrm{H}_{2} \mathrm{O}$ and $\mathrm{CH}_{3} \mathrm{OH}$ masers (Braz \& Epchtein 1983; Caswell \& Haynes 1987; Caswell 1998; Caswell et al. 1989; Caswell et al. 1995) - and their good correlation with massive molecular clumps (see e.g. Fig. 3) suggests that star formation within NGC 3603 is still going on.

In Fig. 9 the total velocity integrated CS (2-1) emission is displayed on top of the central quarter of the WFI image presented in Fig. 1. As one can immediately deduce from the distribution of the molecular gas, star formation in NGC 3603 does not only propagate from the north to the south of the 
OB cluster. There is also strong evidence for significant star formation activity in its far north, in particular in association with the massive molecular clump MM11. The NGC 3603 OB cluster appears to be located rather in the middle than on the edge of the whole scenario. Together with the fact that (so far) no indications for ancient supernova explosions, like SN remnants or shells of swept-up interstellar material, are found within the $\mathrm{H}$ II region, this might indicate that the cluster represents the first generation of stars formed within NGC 3603.

\subsection{Star formation efficiency and gas removal time}

A crucial parameter for the understanding of a star forming region is its star formation efficiency which is defined as the ratio of the total stellar mass to the total stellar + gas mass, i.e.

$\mathrm{SFE}=\frac{\mathcal{M}_{\text {stars }}}{\mathcal{M}_{\text {stars }}+\mathcal{M}_{\text {gas }}^{\text {mol }}+\mathcal{M}_{\text {gas }}^{\text {ion }}}$.

Given the age of the stars, from optical or infrared photometry or spectroscopy, one can also determine the star formation rate of the region:

$\mathrm{SFR}=\frac{\mathcal{M}_{\text {stars }}}{t_{\text {stars }}}$.

And by dividing the total gas mass by the star formation rate one gets an estimate for the gas removal time:

$\tau=1.5 \frac{\mathcal{M}_{\mathrm{gas}}}{\mathrm{SFR}}$,

where the factor 1.5 takes into account gas that is fed from massive stars back to the cloud (e.g. by stellar winds and supernovae; Sandage 1986).

In order to calculate the star formation parameters of NGC 3603 we consider the content of stars, molecular gas and ionized material within a circular area of radius $5 \mathrm{pc}\left(\sim 150^{\prime \prime}\right)$ centered on the OB cluster. Thus, we will include most of the cluster stars (Nürnberger \& Petr-Gotzens 2002) as well as the molecular and ionized material associated with the clumps MM 1, MM 2, MM 6 and MM7. The stellar mass of the cluster is at least $4 \times 10^{3} \mathcal{M}_{\odot}$ (Moffat et al. 1985; Eisenhauer et al. 1998; Brandner et al. 2000). As mentioned above most of the cluster members are $<3$ Myr old (Melnick et al. 1989; Hofmann et al. 1995; Eisenhauer et al. 1998; Brandl et al. 1999). For a $r^{-2}$ density profile a virial gas mass of $5.5 \times 10^{3} \mathcal{M}_{\odot}$ is derived for MM 1, MM 2, MM 6 and MM 7 using the CS (3-2) data set (see Table 2). To estimate the mass of the ionized material, we follow Goss \& Radhakrishnan $(1969,1970)$ and adopt $17 \%$ (properly scaled to a radius of $5 \mathrm{pc}$ ) of their $\mathrm{H}$ II mass, i.e. $2.4 \times 10^{3} \mathcal{M}_{\odot}$, which is consistent with the value given by Retallack \& Goss 1980).

Based on these considerations, we obtain the following parameters: SFE $\gtrsim 30 \%$, SFR $\gtrsim 1.3 \times 10^{-3} \mathcal{M}_{\odot} \mathrm{yr}^{-1}$ and $\tau \sim 6 \mathrm{Myr}$. Hence, the age of the cluster is approximately half of the gas removal time. These values appear reasonable given the fact that the radius of the wind-driven cavity - which is mostly void of interstellar material (Balick et al. 1980; Clayton 1986, $1990)$ - is about $2.5 \mathrm{pc}$, i.e. half the radius $(5 \mathrm{pc}$ ) considered for the above mass estimations.

\section{Summary}

Large scale CS (2-1) and CS (3-2) mapping of the Galactic starburst region NGC 3603 allowed us to investigate the spatial distribution and the kinematic structure of the dense gas in the giant molecular cloud from which the starburst cluster originated. The main results of our study are the following:

- In both CS transitions we have identified 13 molecular clumps. At least 5 clumps exhibit two velocity components, probably indicating two physically separated clumps and being reminiscent of complex gas kinematics within the $\mathrm{H}$ II region. From the average of the line peak velocities $\left(14.2 \pm 1.6 \mathrm{~km} \mathrm{~s}^{-1}\right)$ we deduce a kinematic distance of $7.7 \pm 0.2 \mathrm{kpc}$ for NGC 3603 .

- On average, the molecular clumps have radii smaller than $\langle\mathcal{R}\rangle \sim 0.8 \pm 0.2 \mathrm{pc}, \quad$ virial masses $\left\langle\mathcal{M}_{\text {vir }}\right\rangle \lesssim$ $(1.0 \pm 0.6) \times 10^{3} \mathcal{M}_{\odot}$ and column densities $\left\langle\mathcal{N}\left(\mathrm{H}_{2}\right)\right\rangle \gtrsim$ $(0.4 \pm 0.2) \times 10^{23} \mathrm{~cm}^{-2}$. Clump MM11 stands out with a 4 times higher mass and column density.

- The CS (3-2)/CS (2-1) ratios suggest that both lines are mostly optically thick. The intensity ratios for MM 1 to MM $4(0.80 \pm 0.08)$ are significantly higher than those for MM 6 to MM $12(0.32 \pm 0.11)$. Hence, the northern clumps appear to have either higher opacities or different excitation conditions than the southern clumps.

- Together with masses for the cluster stars and the ionized material taken from the literature, our measurements provide a lower limit for the star formation efficiency, $\gtrsim 30 \%$, in the center $(r \sim 5 \mathrm{pc})$ of the $\mathrm{NGC} 3603 \mathrm{H}$ II region.

- Assuming an age $<3 \mathrm{Myr}$ for the OB cluster and a star formation rate $\gtrsim 1.3 \times 10^{-3} \mathcal{M}_{\odot} \mathrm{yr}^{-1}$, the derived time scale for gas removal $(\tau \sim 6 \mathrm{Myr})$ is consistent with the fact that a cavity of radius $2.5 \mathrm{pc}$, centered on the OB cluster, has been blown free of interstellar material. The adjacent clump MM 1 appears to constitute the head of a prominent pillar, which still becomes dispersed by ionizing radiation and stellar winds originating from the massive stars of the cluster.

- The association of several molecular clumps with near and mid infrared sources as well as with $\mathrm{OH}, \mathrm{H}_{2} \mathrm{O}$ and $\mathrm{CH}_{3} \mathrm{OH}$ masers suggests ongoing formation of massive stars.

Acknowledgements. We are grateful to Thomas Stanke who has kindly obtained the WFI data of NGC 3603. Dennis Downes is thanked for careful reading of and providing valuable comments on an early version of the manuscript. We are indebted to an anonymous referee who greatly helped to correct a nasty error and to improve the consistency of the paper. This research has been supported by the Deutsche Forschungsgemeinschaft (DFG) in the framework of its "Physics of Star Formation" program under grants Yo 5/23-1 and Zi 242/22-2. We also acknowledge support by IRAM, Grenoble (DEAN) and by Chilean Centro de Astrofísica FONDAP No. 15010003 (LB).

\section{References}

Balick, B., Boeshaar, G. O., \& Gull, T. R. 1980, ApJ, 242, 584 Blitz, L. 1993, in Protostars and Planets III, ed. E. H. Levy, \& J. I. Lunine (Univ. of Arizona Press), 125 
Blitz, L., \& Williams, J. P. 1999, in The Origin of Stars and Planetary Systems, ed. C. J. Lada, \& N. D. Kylafis, NATO Sci. Ser. C, 540, 3 Brandl, B., Brandner, W., Eisenhauer, F., et al. 1999, A\&A, 352, L69

Brandner, W., Grebel, E. K., Chu, Y.-H., \& Weis, K. 1997a, ApJ, 475, L45

Brandner, W., Chu, Y.-H., Eisenhauer, F., Grebel, E. K., \& Points, S. D. $1997 b$, ApJ, 489, L153

Brandner, W., Grebel, E. K., Chu, Y.-H., et al. 2000, AJ, 119, 292

Braz, M. A., \& Epchtein, N. 1983, A\&AS, 54, 167

Bronfman, L., Casassus, S., May, J., \& Nyman, L.-Å. 2000, A\&A, 358,521

Caswell, J. L. 1998, MNRAS, 297, 215

Caswell, J. L., \& Haynes, R. F. 1987, Aust. J. Phys., 40, 215

Caswell, J. L., Batchelor, R. A., Forster, J. R., \& Wellington, K. J. 1989, Aust. J. Phys., 42, 331

Caswell, J. L., Vaile, R. A., Ellingsen, S. P., Whiteoak, J. B., \& Norris, R. P. 1995, MNRAS, 272, 96

Clayton, C. A. 1986, MNRAS, 219, 895

Clayton, C. A. 1990, MNRAS, 246, 712

Crowther, P. A., \& Dessart, L. 1998, MNRAS, 296, 622

De Pree, C. G., Nysewander, M. C., \& Goss, W. M. 1999, AJ, 117, 2902

Drissen, L., Moffat, A. F. J., Walborn, N. R., \& Shara, M. M. 1995, AJ, 110, 2235

Eisenhauer, F., Quirrenbach, A., Zinnecker, H., \& Genzel, R. 1998, ApJ, 498, 278

Elmegreen, B. G., Efremov, Y. N., Pudritz, R. E., \& Zinnecker, H. 2000, in Protostars and Planets IV, ed. V. Mannings, A. P. Boss, \& S. S. Russell, 179

Fich, M., Blitz, L., \& Stark, A. A. 1989, ApJ, 342, 272

Frogel, J. A., Persson, S. E., \& Aaronson, M. 1977, ApJ, 213, 723

Georgelin, Y. M., Russeil, D., Amram, P., et al. 2000, A\&A, 357, 308

Goss, W. M., \& Radhakrishnan, V. 1969, ApL, 4, 199

Goss, W. M., Radhakrishnan, V., Brooks, J. W., \& Murray, J. D. 1972 , ApJS, 24, 123

Grabelsky, D. A., Cohen, R. S., Bronfman, L., Thaddeus, P., \& May, J. 1987, ApJ, 315, 122

Grabelsky, D. A., Cohen, R. S., Bronfman, L., \& Thaddeus, P. 1988, ApJ, 331, 181

Hillenbrand, L. A. 1997, AJ, 113, 1733

Hillenbrand, L. A., \& Hartmann, L. W. 1998, ApJ, 492, 540

Hofmann, K.-H., Seggewiess, W., \& Weigelt, G. 1995, A\&A, 300, 403

Hollenbach, D., Johnston, D., Lizano, S., \& Shu, F. H. 1994, ApJ, 428, 654

Kennicutt, R. C. 1984, ApJ, 287, 116

Lada, E. A., Strom, K. M., \& Myers, P. C. 1993, in Protostars and Planets III, ed. E. H. Levy, \& J. I. Lunine, 245

Lee, C. W., Myers, P. C., \& Tafalla, M. 1999, ApJ, 526, 788

MacLaren, I., Richardson, K. M., \& Wolfendale, A. W. 1988, ApJ, 333,821

Mardones, D., Myers, P. C., Tafalla, M., et al. 1997, ApJ, 489, 719

Melnick, J. 1989, The Messenger, 57, 4
Melnick, J., Tapia, M., \& Terlevich, R. 1989, A\&A, 213, 89

Moffat, A. F. J. 1974, A\&A, 35, 315

Moffat, A. F. J. 1983, A\&A, 124, 273

Moffat, A. F. J., Drissen, L., \& Shara, M. M. 1994, ApJ, 436, 183

Moffat, A. F. J., Seggewiss, W., \& Shara, M. M. 1985, ApJ, 295, 109

Mücke, A., Koribalski, B., Moffat, A. F. J., Corcoran, M. F., \& Stevens, I. R. 2002, ApJ, 571, 366

Myers, P. C., Mardones, D., Tafalla, M., Williams, J. P., \& Wilner, D. J. 1996, ApJ, 465, L133

Nürnberger, D. E. A. 2002a, in Hot Star Workshop III: The Earliest Phases of Massive Star Birth, ed. P. A. Crowther, ASP Conf. Ser., 267, 127

Nürnberger, D. E. A. 2002b, Ph.D. Thesis, University Würzburg, in preparation

Nürnberger, D. E. A., \& Petr-Gotzens, M. G. 2002, A\&A, 382, 537

Nürnberger, D. E. A., \& Stanke, Th. 2002, A\&A, submitted

Nürnberger, D., Bronfman, L., Petr-Gotzens, M., \& Stanke, Th. 2002, in The Origin of Stars and Planets: The VLT View, ed. J. Alves, $\&$ M. McCaughrean, ESO Astrophys. Symp. Ser., in press

Nürnberger, D., Durand, S., Köppen, J., et al. 2001, A\&A, 377, 241

Pandey, A. K., Ogura, K., \& Sekiguchi, K. 2000, PASJ, 52, 847

Persi, P., Tapia, M., Roth, M., \& Ferrari-Toniolo, M. 1985, A\&A, 144, 275

Pound, M. 1998, ApJ, 493, L113

Retallack, D. S., \& Goss, W. M. 1980, MNRAS, 193, 261

Roth, M., Tapia, M., Ruiz, M. T., Persi, P., \& Ferrari-Toniolo, M. 1987, in Star Forming Regions, ed. M. Peimbert, \& J. Jugaku, IAU Symp., 115, 182

Sandage, A. 1986, A\&A, 161, 89

Shobbrook, R. R., \& Lynga, G. 1994, MNRAS, 269, 857

Stark, A. A, \& Brand, J. 1989, ApJ, 339, 763

Tapia, M. 1981, MNRAS, 197, 949

Van den Bergh, S. 1978, A\&A, 63, 275

Walborn, N. R. 1973, ApJ, 182, L21

Williams, J. P, \& Blitz, L. 1998, ApJ, 494, 657

Williams, J. P, \& McKee, C. F. 1997, ApJ, 476, 166

Williams, J. P, \& Myers, P. C. 1999, ApJ, 511, 208

Williams, J. P, Blitz, L., \& McKee, C. F. 2000, in Protostars and Planets IV, ed. V. Mannings, A. P. Boss, \& S. S. Russell (Univ. of Arizona Press), 97

Williams, J. P, Blitz, L., \& Stark, A. A. 1995, ApJ, 451, 252

Wilson, T. L., Mauersberger, R., Gensheimer, P. D., Muders, D., \& Bieging, J. H. 1999, ApJ, 525, 343

Wolfire, M. G., \& Cassinelli, J. P. 1987, ApJ, 319, 850

Yorke, H. W., \& Krügel, E. 1977, A\&A, 54, 183

Yorke, H. W., \& Welz, A. 1993, in Star Formation, Galaxies and the Interstellar Medium, ed. J. Franco, F. Ferrini, \& G. Tenorio-Tagle (Cambridge Univ. Press), 239

Zinchenko, I., Mattila, K., \& Toriseva, M. 1995, A\&AS, 111, 95

Zinnecker, H., McCaughrean, M. J., \& Wilking, B. A. 1993, in Protostars and Planets III, ed. E. H. Levy, \& J. I. Lunine, 429 